\title{
Complete Elliptic Integrals Resulting from Infinite Integrals of Bessel Functions*
}

\author{
Shigehiko Okui**
}

(June 12, 1974)

\begin{abstract}
Infinite integrals of Bessel and modified Bessel functions reducible to complete elliptic integrals are compiled. These formulas are of great use in solving problems of applied mathematics, physics and engineering.
\end{abstract}

Key words: Applied mathematics; Bessel functions; complete elliptic integrals; engineering; infinite integrals; modified Bessel functions; physics; signal statistics.

AMS Subject Classification: 33A25, 33A40, 44A20.

*An invited paper.

**Present address: Kobe City Technical College, Tarumi-ku, Kobe, Japan.

\section{Introduction}

This table, which is an outgrowth of the author's experience in the theoretical study of signal statistics, contains infinite integrals of Bessel and modified Bessel functions reducible to complete elliptic integrals. ${ }^{1}$ The formulas listed below are those important in applications and all results are expressed in conviently compact forms.

The materials were first extracted from the author's own memorandum which were then thoroughly augmented and rearranged in the present form by scrutinizing various published books and papers.

The parameters used in this table are usually positive real and notations occurring several times on a section are explained at the top of the section.

\section{Integrands Involving Bessel Functions of the First Kind}

$$
\begin{aligned}
& \text { 2.1. } k^{2}=\frac{\sqrt{p^{2}+a^{2}}-p}{2 \sqrt{p^{2}+a^{2}}} \\
& \int_{0}^{\infty} e^{-p x^{2}} J_{0}\left(a x^{2}\right) d x=\frac{\sqrt{1-2 k^{2}}}{\sqrt{\pi p}} K(k)
\end{aligned}
$$

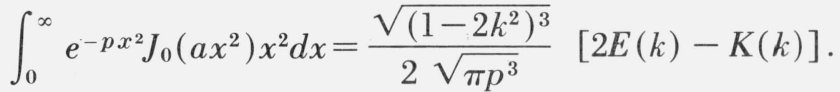

$$
\begin{aligned}
& \text { OL } 119 \text { (13.1) }
\end{aligned}
$$

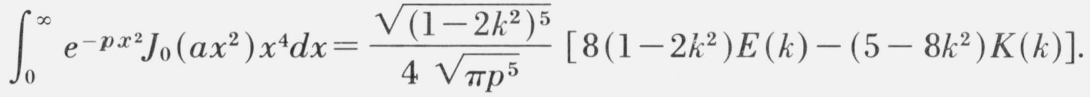

'Including Bierens de Haan, D., Nouvelles Tables d'Intégrales Définies (Leide, Amsterdam 1867), there exists a considerable literature on complete elliptic integrals involving algebraic, trigonometric, hyperbolic or logarithmic integrands. 


$$
\int_{0}^{\infty} e^{-p x^{2}} J_{0}\left(a x^{2}\right) x^{6} d x=\frac{\sqrt{\left(1-2 k^{2}\right)^{7}}}{8 \sqrt{\pi p^{7}}}\left[2\left(23-128 k^{2}+128 k^{4}\right) E(k)-\left(31-144 k^{2}+128 k^{4}\right) K(k)\right] .
$$

$$
\int_{0}^{\infty} e^{-p x^{2}} J_{1}\left(a x^{2}\right) d x=\frac{\sqrt{1-2 k^{2}}}{k \sqrt{\pi p\left(1-k^{2}\right)}}\left[E(k)-\left(1-k^{2}\right) K(k)\right] .
$$

$$
\int_{0}^{\infty} e^{-p x^{2}} J_{1}\left(a x^{2}\right) x^{2} d x=\frac{\sqrt{\left(1-2 k^{2}\right)^{3}}}{2 k \sqrt{\pi p^{3}\left(1-k^{2}\right)}}\left[\left(1-k^{2}\right) K(k)-\left(1-2 k^{2}\right) E(k)\right] .
$$

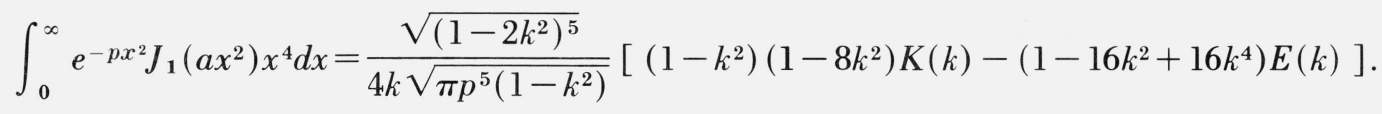

$$
\int_{0}^{\infty} e^{-p x^{2}} J_{1}\left(a x^{2}\right) x^{-2} d x=\frac{2 \sqrt{p}}{3 k \sqrt{\pi\left(1-k^{2}\right)\left(1-2 k^{2}\right)}}\left[\left(1-k^{2}\right) K(k)-\left(1-2 k^{2}\right) E(k)\right] .
$$$$
\int_{0}^{\infty} e^{-p x^{2}} J_{2}\left(a x^{2}\right) d x=\frac{\sqrt{1-2 k^{2}}}{3 k^{2}\left(1-k^{2}\right) \sqrt{\pi p}}\left[\left(1-k^{2}\right)\left(2-3 k^{2}\right) K(k)-2\left(1-2 k^{2}\right) E(k)\right] .
$$

$$
\int_{0}^{\infty} e^{-p x^{2}} J_{2}\left(a x^{2}\right) x^{2} d x=\frac{\sqrt{\left(1-2 k^{2}\right)^{3}}}{2 k^{2}\left(1-k^{2}\right) \sqrt{\pi p^{3}}}\left[2\left(1-k^{2}+k^{4}\right) E(k)-\left(1-k^{2}\right)\left(2-k^{2}\right) K(k)\right] .
$$

$$
\int_{0}^{\infty} e^{-p x^{2}} J_{2}\left(a x^{2}\right) x^{4} d x=\frac{\sqrt{\left(1-2 k^{2}\right)^{5}}}{4 k^{2}\left(1-k^{2}\right) \sqrt{\pi p^{5}}}\left[\left(2+5 k^{2}-8 k^{4}\right)\right.
$$

$$
\left.\times\left(1-k^{2}\right) K(k)-2\left(1-2 k^{2}\right)\left(1+4 k^{2}-4 k^{4}\right) E(k)\right] .
$$

$$
\int_{0}^{\infty} e^{-p x^{2}} J_{2}\left(a x^{2}\right) x^{6} d x=\frac{\sqrt{\left(1-2 k^{2}\right)^{7}}}{8 k^{2}\left(1-k^{2}\right) \sqrt{\pi p^{7}}}\left[\left(2+15 k^{2}-144 k^{4}+128 k^{6}\right)\right.
$$

$$
\left.\times\left(1-k^{2}\right) K(k)-2\left(1+7 k^{2}-135 k^{4}+256 k^{6}-128 k^{8}\right) E(k)\right] .
$$

$$
\begin{aligned}
& \int_{0}^{\infty} e^{-p x^{2}} J_{2}\left(a x^{2}\right) x^{-2} d x=\frac{2 \sqrt{p}}{15 k^{2}\left(1-k^{2}\right) \sqrt{\pi\left(1-2 k^{2}\right)}} \\
& \quad \times\left[2\left(1-k^{2}+k^{4}\right) E(k)-\left(1-k^{2}\right)\left(2-k^{2}\right) K(k)\right] .
\end{aligned}
$$

$$
\begin{aligned}
\int_{0}^{\infty} e^{-p x^{2}} J_{3}\left(a x^{2}\right) d x=\frac{\sqrt{1-2 k^{2}}}{15 k^{3} \sqrt{\pi p\left(1-k^{2}\right)^{3}}}\left[\left(8-23 k^{2}+23 k^{4}\right) E(k)\right. & \\
& \left.-\left(1-k^{2}\right)\left(8-19 k^{2}+15 k^{4}\right) K(k)\right] .
\end{aligned}
$$

$$
\begin{aligned}
\int_{0}^{\infty} e^{-p x^{2}} J_{3}\left(a x^{2}\right) x^{2} d x=\frac{\sqrt{\left(1-2 k^{2}\right)^{3}}}{6 k^{3} \sqrt{\pi p^{3}\left(1-k^{2}\right)^{3}}} & {\left[\left(8-15 k^{2}+3 k^{4}\right)\right.} \\
& \left.\times\left(1-k^{2}\right) K(k)-\left(8-19 k^{2}+9 k^{4}-6 k^{6}\right) E(k)\right] .
\end{aligned}
$$




$$
\begin{aligned}
\int_{0}^{\infty} e^{-p x^{2}} J_{3}\left(a x^{2}\right) x^{-2} d x= & \frac{2 \sqrt{p}}{105 k^{3} \sqrt{\pi\left(1-k^{2}\right)^{3}\left(1-2 k^{2}\right)}}\left[\left(1-k^{2}\right)\right. \\
& \left.\times\left(8-15 k^{2}+3 k^{4}\right) K(k)-\left(8-19 k^{2}+9 k^{4}-6 k^{6}\right) E(k)\right] .
\end{aligned}
$$

2.2. $k_{1}^{2}=\frac{1}{2}\left(1-\sqrt{1-a^{2} \gamma^{2}}\right), \quad k_{2}^{2}=\frac{1}{2}\left(1-\sqrt{1-b^{2} \gamma^{2}}\right)$,

$$
\gamma^{2}=\frac{2}{p^{2}+a^{2}+b^{2}+\sqrt{\left(p^{2}+a^{2}+b^{2}\right)^{2}-4 a^{2} b^{2}}}
$$

$$
\int_{0}^{\infty} e^{-p x^{2}} J_{0}\left(a x^{2}\right) J_{0}\left(b x^{2}\right) d x=\frac{2 \sqrt{\gamma}}{\sqrt{\pi^{3}}} K\left(k_{1}\right) K\left(k_{2}\right) .
$$

$\int_{0}^{\infty} e^{-p x^{2}} J_{1}\left(a x^{2}\right) J_{0}\left(b x^{2}\right) d x=\frac{2 \sqrt{\gamma}}{k_{1} \sqrt{\pi^{3}\left(1-k_{1}^{2}\right)}}$

$$
\times\left[E\left(k_{1}\right)-\left(1-k_{1}^{2}\right) K\left(k_{1}\right)\right]\left[2 E\left(k_{2}\right)-K\left(k_{2}\right)\right] .
$$

$$
\begin{aligned}
\int_{0}^{\infty} e^{-p x^{2}} J_{1}\left(a x^{2}\right) J_{1}\left(b x^{2}\right) d x= & \frac{2 \sqrt{\gamma}}{3 k_{1} k_{2} \sqrt{\pi^{3}\left(1-k_{1}^{2}\right)\left(1-k_{2}^{2}\right)}} \\
& \times\left[\left(1-2 k_{1}^{2}\right) E\left(k_{1}\right)-\left(1-k_{1}^{2}\right) K\left(k_{1}\right)\right] \\
& \times\left[\left(1-2 k_{2}^{2}\right) E\left(k_{2}\right)-\left(1-k_{2}^{2}\right) K\left(k_{2}\right)\right] .
\end{aligned}
$$

$$
\begin{aligned}
\int_{0}^{\infty} e^{-p x^{2} J_{2}\left(a x^{2}\right) J_{0}\left(b x^{2}\right) d x}=\frac{2 \sqrt{\gamma}}{9 k_{1}^{2}\left(1-k_{1}^{2}\right) \sqrt{\pi^{3}}} & \\
\times & \times\left[\left(1-k_{1}^{2}\right)\left(2-3 k_{1}^{2}\right) K\left(k_{1}\right)-2\left(1-2 k_{1}^{2}\right) E\left(k_{1}\right)\right] \\
& \times\left[8\left(1-2 k_{2}\right) E\left(k_{2}\right)-\left(5-8 k_{2}^{2}\right) K\left(k_{2}\right)\right] .
\end{aligned}
$$

$$
\begin{aligned}
& \int_{0}^{\infty} e^{-p x^{2} J_{2}\left(a x^{2}\right) J_{1}\left(b x^{2}\right) d x}= \frac{2 \sqrt{\gamma}}{15 k_{1}^{2} k_{2}\left(1-k_{1}^{2}\right) \sqrt{\pi^{3}\left(1-k_{2}^{2}\right)}} \\
& \times\left[2\left(1-k_{1}^{2}+k_{1}^{4}\right) E\left(k_{1}\right)-\left(1-k_{1}^{2}\right)\left(2-k_{1}^{2}\right) K\left(k_{1}\right)\right] \\
& \quad \times\left[\left(1-k_{2}^{2}\right)\left(1-8 k_{2}^{2}\right) K\left(k_{2}\right)-\left(1-16 k_{2}^{2}+16 k_{2}^{4}\right) E\left(k_{2}\right)\right] .
\end{aligned}
$$

$$
\begin{aligned}
\int_{0}^{\infty} e^{-p x^{2}} J_{2}\left(a x^{2}\right) J_{2}\left(b x^{2}\right) d x=\frac{2 \sqrt{\gamma}}{105 k_{1}^{2} k_{2}^{2}\left(1-k_{1}^{2}\right)\left(1-k_{2}^{2}\right) \sqrt{\pi^{3}}} \\
\quad \times\left[\left(1-k_{1}^{2}\right)\left(2+5 k_{1}^{2}-8 k_{1}^{4}\right) K\left(k_{1}\right)-2\left(1-2 k_{1}^{2}\right)\left(1+4 k_{1}^{2}-4 k_{1}^{4}\right) E\left(k_{1}\right)\right] \\
\quad \times\left[\left(1-k_{2}^{2}\right)\left(2+5 k_{2}^{2}-8 k_{2}^{4}\right) K\left(k_{2}\right)-2\left(1-2 k_{2}^{2}\right)\left(1+4 k_{2}^{2}-4 k_{2}^{4}\right) E\left(k_{2}\right)\right] .
\end{aligned}
$$




$$
\begin{aligned}
& \text { 2.3. } k^{2}=\frac{a^{2}}{p^{2}+a^{2}} \\
& \int_{0}^{\infty} e^{-2 p x} J_{0}^{2}(a x) x^{2} d x=\frac{k}{4 \pi p^{2} a}\left[\left(3-2 k^{2}\right) E(k)-\left(1-k^{2}\right) K(k)\right] . \\
& \int_{0}^{\infty} e^{-2 p x} J_{1}^{2}(a x) x^{2} d x=\frac{k}{4 \pi p^{2} a}\left[\left(1-k^{2}\right) K(k)-\left(1-2 k^{2}\right) E(k)\right] . \quad \text { BY } 251(561.08)^{2} \\
& \int_{0}^{\infty} e^{-2 p x} J_{1}^{2}(a x) x^{-2} d x=\frac{4 a}{3 \pi k^{3}}\left[\left(1-k^{2}\right) K(k)-\left(1-2 k^{2}\right) E(k)\right]-p . \\
& \int_{0}^{\infty} e^{-2 p x} J_{2}^{2}(a x) x d x=\frac{1}{2 \pi p a k^{3}}\left[\left(16-16 k^{2}+k^{4}\right) E(k)-8\left(1-k^{2}\right)\left(2-k^{2}\right) K(k)\right] . \\
& \int_{0}^{\infty} e^{-2 p x} J_{2}^{2}(a x) x^{-2} d x=\frac{4 a}{15 \pi k^{5}}\left[\left(1-k^{2}\right)\left(4+3 k^{2}\right) K(k)-\left(4+k^{2}-6 k^{4}\right) E(k)\right]-\frac{p}{2} . \\
& \text { 2.4. } \quad k^{2}=\frac{a^{2}}{p^{2}+a^{2}} \\
& \int_{0}^{\infty} e^{-2 p x} J_{2}(a x) J_{1}(a x) x^{-1} d x=\frac{2}{3 \pi k^{3}}\left[4\left(1-k^{2}\right) K(k)-\left(4-5 k^{2}\right) E(k)\right]-\frac{p}{a} . \\
& \int_{0}^{\infty} e^{-2 p x} J_{3}(a x) J_{1}(a x) d x=\frac{1}{3 \pi a k^{3}}\left[\left(32-38 k^{2}+3 k^{4}\right) K(k)-2\left(16-23 k^{2}\right) E(k)\right]-\frac{4 p}{a^{2}} . \\
& \int_{0}^{\infty} e^{-2 p x} J_{3}(a x) J_{2}(a x) x^{-1} d x=\frac{2}{15 \pi k^{5}}\left[4\left(1-k^{2}\right)\left(8+k^{2}\right) K(k)-\left(32-12 k^{2}-23 k^{4}\right) E(k)\right]-\frac{p}{a} .
\end{aligned}
$$

$$
\begin{gathered}
\text { 2.5. } \boldsymbol{k}^{2}=\frac{\mathbf{4 a b}}{\mathbf{p}^{2}+(\mathbf{a}+\boldsymbol{b})^{2}} \\
\int_{0}^{\infty} e^{-p x} J_{0}(a x) J_{0}(b x) d x=\frac{k}{\pi \sqrt{a b}} K(k) . \quad \text { BY } 248(560.01) \\
\int_{0}^{\infty} e^{-p x} J_{0}(a x) J_{0}(b x) x d x=\frac{p k^{3}}{4 \pi\left(1-k^{2}\right) \sqrt{(a} \frac{\bar{b})^{3}}{}} E(k) . \quad \text { LU } 316 \text { (19) }
\end{gathered}
$$


$\int_{0}^{\infty} e^{-p x} J_{1}(a x) J_{0}(b x) x d x=\frac{k}{8 \pi\left(1-k^{2}\right) a \sqrt{(a b)^{3}}}\left[k^{2}\left(a^{2}-b^{2}-p^{2}\right) E(k)+4 a b\left(1-k^{2}\right) K(k)\right]$.

LU 317 (21)

$$
\int_{0}^{\infty} e^{-p x} J_{1}(a x) J_{1}(b x) d x=\frac{1}{\pi k \sqrt{a b}}\left[\left(2-k^{2}\right) K(k)-2 E(k)\right] .
$$

BY $249(560.02)$

$$
\int_{0}^{\infty} e^{-p x} J_{1}(a x) J_{1}(b x) x d x=\frac{p k}{4 \pi\left(1-k^{2}\right) \sqrt{(a b)^{3}}}\left[\left(2-k^{2}\right) E(k)-2\left(1-k^{2}\right) K(k)\right] .
$$

LU 316 (20)

$$
\int_{0}^{\infty} e^{-p x} J_{2}(a x) J_{2}(b x) d x=\frac{1}{3 \pi k^{3} \sqrt{a b}}\left[\left(4-k^{2}\right)\left(4-3 k^{2}\right) K(k)-8\left(2-k^{2}\right) E(k)\right] .
$$

BY 248 (560.03)

$$
\begin{aligned}
& \int_{0}^{\infty} e^{-p x} J_{3}(a x) J_{3}(b x) d x=\frac{1}{15 \pi k^{5} \sqrt{a b}}[\left(128-128 k^{2}+15 k^{4}\right) \\
&\left.\times\left(2-k^{2}\right) K(k)-2\left(128-128 k^{2}+23 k^{4}\right) E(k)\right] . \\
& \int_{0}^{\infty} e^{-p x} J_{4}(a x) J_{4}(b x) d x=\frac{1}{105 \pi k^{7} \sqrt{a b}}\left[\left(6144-12288 k^{2}\right.\right. \\
&+8000 k^{4}-\left.\left.1856 k^{6}+105 k^{8}\right) K(k)-32\left(2-k^{2}\right)\left(96-96 k^{2}+11 k^{4}\right) E(k)\right] .
\end{aligned}
$$

$$
2.6 \quad k^{2}=\frac{4 a b}{p^{2}+(a+b)^{2}}=\sin ^{2} \alpha, \quad \sin \beta=\frac{p}{\sqrt{p^{2}+(a-b)^{2}}}
$$

\section{$\Lambda_{0}(\alpha, \beta)$ is Heuman's Lambda function. ${ }^{3}$}

$$
\begin{aligned}
\int_{0}^{\infty} e^{-p x} J_{1}(a x) J_{0}(b x) d x & =-\frac{p k}{2 \pi a \sqrt{a b}} K(k)-\frac{1}{2 a} \Lambda_{0}(\alpha, \beta)+\frac{1}{a}, \quad a>b ; \\
& =-\frac{p k}{2 \pi a^{2}} K(k)+\frac{1}{2 a}, \quad a=b ; \\
& =-\frac{p k}{2 \pi a \sqrt{a b}} K(k)+\frac{1}{2 a} \Lambda_{0}(\alpha, \beta), \quad a<b .
\end{aligned}
$$

LU $317(22)$

$$
\begin{aligned}
\int_{0}^{\infty} e^{-p x} J_{1}(a x) J_{0}(b x) x^{-1} d x & =\frac{2 \sqrt{a b}}{\pi a k} E(k)+\frac{k\left(a^{2}-b^{2}\right)}{2 \pi a \sqrt{a b}} K(k)+\frac{p}{2 a} \Lambda_{0}(\alpha, \beta)-\frac{p}{a}, \quad a>b ; \\
& =\frac{2}{\pi k} E(k)-\frac{p}{2 a}, \quad a=b ;
\end{aligned}
$$

${ }^{3}$ In connection with the Heuman's Lambda function the reader should consult BY pp. 35-37. 


$$
=\frac{2 \sqrt{a b}}{\pi a k} E(k)+\frac{k\left(a^{2}-b^{2}\right)}{2 \pi a \sqrt{a b}} K(k)-\frac{p}{2 a} \Lambda_{0}(\alpha, \beta), \quad a<b .
$$

$$
\begin{aligned}
\int_{0}^{\infty} e^{-p x} J_{1}(a x) J_{1}(b x) x^{-1} d x= & \frac{p}{\pi k \sqrt{a b}} E(k)-\frac{p k\left(p^{2}+2 a^{2}+2 b^{2}\right)}{4 \pi \sqrt{(a b)^{3}}} K(k)+\frac{\left(a^{2}-b^{2}\right)}{4 a b} \Lambda_{0}(\alpha, \beta) \\
& +\frac{b}{2 a}, \quad a>b ; \\
= & \frac{p}{\pi a k} E(k)-\frac{p k\left(p^{2}+4 a^{2}\right)}{4 \pi a^{3}} K(k)+\frac{1}{2}, \quad a=b ; \\
= & \frac{p}{\pi k \sqrt{a b}} E(k)-\frac{p k\left(p^{2}+2 a^{2}+2 b^{2}\right)}{4 \pi \sqrt{(a b)^{3}}} K(k)-\frac{\left(a^{2}-b^{2}\right)}{4 a b} \Lambda_{0}(\alpha, \beta) \\
& +\frac{a}{2 b}, \quad a<b .
\end{aligned}
$$

$$
\begin{aligned}
\int_{0}^{\infty} e^{-p x} J_{2}(a x) J_{0}(b x) d x & =\frac{4 \sqrt{a b}}{\pi a^{2} k} E(k)-\frac{k b^{2}}{\pi a^{2} \sqrt{a b}} K(k)+\frac{p}{a^{2}} \Lambda_{0}(\alpha, \beta)-\frac{2 p}{a^{2}}, \quad a>b ; \\
& =\frac{4}{\pi a k} E(k)-\frac{k}{\pi a} K(k)-\frac{p}{a^{2}}, \quad a=b ; \\
& =\frac{4 \sqrt{a b}}{\pi a^{2} k} E(k)-\frac{k b^{2}}{\pi a^{2} \sqrt{a b}} K(k)-\frac{p}{a^{2}} \Lambda_{0}(\alpha, \beta), \quad a<b .
\end{aligned}
$$

$$
\begin{aligned}
\int_{0}^{\infty} e^{-p x} J_{2}(a x) J_{0}(b x) x d x= & -\frac{p k}{\pi a^{2} \sqrt{a b}} K(k)-\frac{p k^{3}}{4 \pi\left(1-k^{2}\right) \sqrt{(a b)^{3}}} E(k)-\frac{1}{a^{2}} \Lambda_{0}(\alpha, \beta) \\
& +\frac{2}{a^{2}}, \quad a>b \\
= & -\frac{p k}{\pi a^{3}} K(k)-\frac{p k^{3}}{4 \pi\left(1-k^{2}\right) a^{3}} E(k)+\frac{1}{a^{2}}, \quad a=b \\
= & -\frac{p k}{\pi a^{2} \sqrt{a b}} K(k)-\frac{p k^{3}}{4 \pi\left(1-k^{2}\right) \sqrt{(a b)^{3}}} E(k) \\
& +\frac{1}{a^{2}} \Lambda_{0}(\alpha, \beta), \quad a<b .
\end{aligned}
$$




$$
\begin{aligned}
\int_{0}^{\infty} e^{-p x} J_{2}(a x) J_{1}(b x) d x= & \frac{2 p}{\pi k a \sqrt{a b}} E(k)-\frac{p k\left(p^{2}+a^{2}+2 b^{2}\right)}{2 \pi a \sqrt{(a b)^{3}}} K(k)-\frac{b}{2 a^{2}} \Lambda_{0}(\alpha, \beta) \\
& +\frac{b}{a^{2}}, \quad a>b ; \\
= & \frac{2 p}{\pi a^{2} k} E(k)-\frac{p k\left(p^{2}+3 a^{2}\right)}{2 \pi a^{4}} K(k)+\frac{1}{2 a}, \quad a=b ; \\
= & \frac{2 p}{\pi k a \sqrt{a b}} E(k)-\frac{p k\left(p^{2}+a^{2}+2 b^{2}\right)}{2 \pi a \sqrt{(a b)^{3}}} K(k)+\frac{b}{2 a^{2}} \Lambda_{0}(\alpha, \beta) \\
& +\frac{1}{b}, \quad a<b .
\end{aligned}
$$

$$
\text { 2.7. } k^{2}=\frac{4 a b}{(a+b)^{2}}
$$

$$
\int_{0}^{\infty} J_{1}(a x) J_{1}(b x) x^{-2} d x=\frac{a+b}{3 \pi a b}\left[\left(a^{2}+b^{2}\right) E(k)-(a-b)^{2} K(k)\right] .
$$

$$
\int_{0}^{\infty} J_{2}(a x) J_{1}(b x) x^{-1} d x=\frac{1}{3 \pi a^{2} b}\left[(a-b)\left(a^{2}+2 b^{2}\right) K(k)-(a+b)\left(a^{2}-2 b^{2}\right) E(k)\right] .
$$

$$
\int_{0}^{\infty} J_{3}(a x) J_{1}(b x) d x=\frac{1}{3 \pi a^{3} b(a+b)}\left[\left(a^{4}+a^{2} b^{2}-8 b^{4}\right) K(k)-(a+b)^{2}\left(a^{2}-8 b^{2}\right) E(k)\right] .
$$

$$
\begin{aligned}
\int_{0}^{\infty} J_{3}(a x) J_{2}(b x) x^{-1} d x=\frac{1}{15 \pi a^{3} b^{2}}\left[( a - b ) \left(2 a^{4}+5 a^{2} b^{2}\right.\right. & \left.+8 b^{4}\right) K(k) \\
& \left.-(a+b)\left(2 a^{4}+3 a^{2} b^{2}-8 b^{4}\right) E(k)\right] .
\end{aligned}
$$

$$
\begin{aligned}
\int_{0}^{\infty} J_{4}(a x) J_{2}(b x) d x=\frac{2}{15 \pi a^{4} b^{2}(a+b)}\left[\left(a^{6}+4 a^{4} b^{2}+4 a^{2} b^{4}-24 b^{6}\right)\right. \\
\\
\left.\times K(k)-(a+b)^{2}\left(a^{4}+4 a^{2} b^{2}-24 b^{4}\right) E(k)\right] .
\end{aligned}
$$

$$
\begin{gathered}
\text { 2.8. } \quad \boldsymbol{k}^{2}=\frac{\boldsymbol{b}-\sqrt{\mathbf{b}^{2}-\mathbf{a}^{2}}}{\mathbf{2 b}}, \quad \mathbf{b}>\boldsymbol{a} \\
\int_{0}^{\infty} J_{0}^{2}(a x) J_{0}(2 b x) d x=\frac{2}{\pi^{2} b}[K(k)]^{2} . \\
\int_{0}^{\infty} J_{0}^{2}(a x) J_{2}(2 b x) d x=\frac{2}{\pi^{2} b}[2 E(k)-K(k)]^{2} . \\
\int_{0}^{\infty} J_{1}^{2}(a x) J_{0}(2 b x) d x=-\frac{8 b}{\pi^{2} a^{2}}\left[E(k)-\left(1-k^{2}\right) K(k)\right]^{2} . \\
\int_{0}^{\infty} J_{1}^{2}(a x) J_{2}(2 b x) d x=\frac{8 b}{3 \pi^{2} a^{2}}\left[\left(1-k^{2}\right) K(k)-\left(1-2 k^{2}\right) E(k)\right]^{2} .
\end{gathered}
$$




$$
\begin{gathered}
\int_{0}^{\infty} J_{2}^{2}(a x) J_{0}(2 b x) d x=\frac{32 b^{3}}{9 \pi^{2} a^{4}}\left[\left(2-3 k^{2}\right)\left(1-k^{2}\right) K(k)-2\left(1-2 k^{2}\right) E(k)\right]^{2} . \\
\int_{0}^{\infty} J_{2}^{2}(a x) J_{2}(2 b x) d x=-\frac{32 b^{3}}{15 \pi^{2} a^{4}}\left[2\left(1-k^{2}+k^{4}\right) E(k)-\left(1-k^{2}\right)\left(2-k^{2}\right) K(k)\right]^{2} . \\
\int_{0}^{\infty} J_{2}^{2}(a x) J_{4}(2 b x) d x=\frac{32 b^{3}}{105 \pi^{2} a^{4}}\left[\left(2+5 k^{2}-8 k^{4}\right)\left(1-k^{2}\right) K(k)\right. \\
\int_{0}^{\infty} J_{3}^{2}(a x) J_{0}(2 b x) d x=-\frac{128 b^{5}}{225 \pi^{2} a^{6}}\left[\left(8-23 k^{2}+23 k^{4}\right) E(k)-\left(1-k^{2}\right)\left(8-19 k^{2}+15 k^{4}\right) K(k)\right]^{2} . \\
\int_{0}^{\infty} J_{3}^{2}(a x) J_{2}(2 b x) d x=\frac{128 b^{5}}{315 \pi^{2} a^{6}}\left[\left(8-15 k^{2}+3 k^{4}\right)\left(1-k^{2}\right) K(k)-\left(8-19 k^{2}\right) E(k)\right]^{2} .
\end{gathered}
$$

$$
\begin{aligned}
& \text { 2.9. } k_{1}^{2}=\frac{a b c d}{\Delta^{2}}, \quad k_{2}^{2}=\frac{\Delta^{2}}{a b c d} \\
& 16 \Delta^{2}=(a+b+c-d)(a+b+d-c)(a+c+d-b)(b+c+d-a) \\
& \int_{0}^{\infty} J_{0}(a x) J_{0}(b x) J_{0}(c x) J_{0}(d x) x d x=\frac{1}{\pi^{2} \Delta} K\left(k_{1}\right), \quad \Delta^{2}>a b c d \\
& =\frac{1}{\pi^{2} \sqrt{a b c d}} K\left(k_{2}\right), \quad \Delta^{2}<a b c d . \quad \text { W A 414 (9) }
\end{aligned}
$$

\section{Integrands Involving Bessel Functions of the Second Kind}

$$
\begin{aligned}
& \text { 3.1. } k^{2}=\frac{\sqrt{p^{2}+a^{2}}+p}{2 \sqrt{p^{2}+a^{2}}} \\
& \int_{0}^{\infty} e^{-p x^{2}} Y_{0}\left(a x^{2}\right) d x=-\frac{\sqrt{2 k^{2}-1}}{\sqrt{\pi p}} K(k)
\end{aligned}
$$

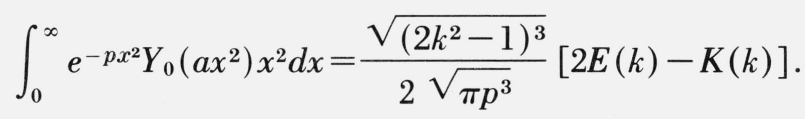

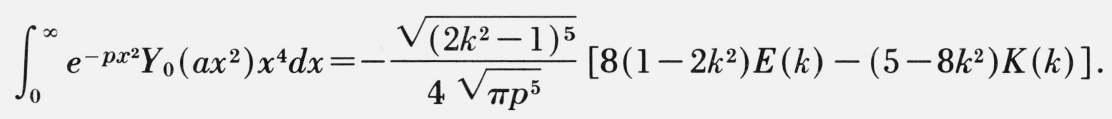

$$
\begin{aligned}
& \int_{0}^{\infty} e^{-p x^{2}} Y_{0}\left(a x^{2}\right) x^{6} d x=\frac{\sqrt{\left(2 k^{2}-1\right)^{7}}}{8 \sqrt{\pi p^{7}}}\left[2\left(23-128 k^{2}+128 k^{4}\right) E(k)-\left(31-144 k^{2}+128 k^{4}\right) K(k)\right] .
\end{aligned}
$$




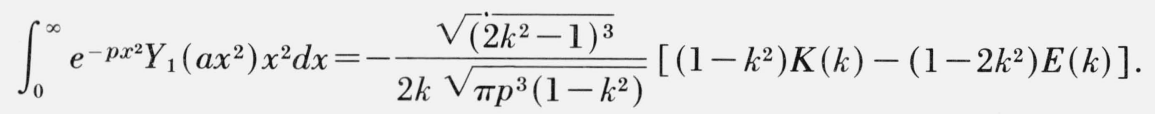

$\int_{0}^{\infty} e^{-p x^{2}} Y_{1}\left(a x^{2}\right) x^{4} d x=\frac{\sqrt{\left(2 k^{2}-1\right)^{5}}}{4 k \sqrt{\pi p^{5}\left(1-k^{2}\right)}}$

$$
\times\left[\left(1-k^{2}\right)\left(1-8 k^{2}\right) K(k)-\left(1-16 k^{2}+16 k^{4}\right) E(k)\right] .
$$

$$
\begin{aligned}
\int_{0}^{\infty} e^{-p x^{2} Y_{1}\left(a x^{2}\right)} x^{6} d x & =-\frac{\sqrt{\left(2 k^{2}-1\right)^{7}}}{8 k \sqrt{\pi p^{7}\left(1-k^{2}\right)}} \\
\times & {\left[\left(3-80 k^{2}+128 k^{4}\right)\left(1-k^{2}\right) K(k)-\left(3-134 k^{2}+384 k^{4}-256 k^{6}\right) E(k)\right] . }
\end{aligned}
$$

$$
\begin{aligned}
& \int_{0}^{\infty} e^{-p x^{2} Y_{2}\left(a x^{2}\right) x^{4} d x=}-\frac{\sqrt{\left(2 k^{2}-1\right)^{5}}}{4 k^{2}\left(1-k^{2}\right) \sqrt{\pi p^{5}}} \\
& \times\left[\left(2+5 k^{2}-8 k^{4}\right)\left(1-k^{2}\right) K(k)-2\left(1-2 k^{2}\right)\left(1+4 k^{2}-4 k^{4}\right) E(k)\right] . \\
& \int_{0}^{\infty} e^{-p x^{2} Y_{2}\left(a x^{2}\right) x^{6} d x}=\frac{\sqrt{\left(2 k^{2}-1\right)^{7}}}{8 k^{2}\left(1-k^{2}\right) \sqrt{\pi p^{7}}} \\
& \quad \times\left[\left(2+15 k^{2}-144 k^{4}+128 k^{6}\right)\left(1-k^{2}\right) K(k)-2\left(1+7 k^{2}-135 k^{4}+256 k^{6}-128 k^{8}\right) E(k)\right] .
\end{aligned}
$$

$$
\text { 3.2. } k^{2}=\frac{p^{2}}{p^{2}+a^{2}}
$$

$$
\begin{gathered}
\int_{0}^{\infty} e^{-2 p x} Y_{0}(a x) J_{0}(a x) d x=-\frac{k}{\pi p} K(k) \cdot \quad \text { OL } 122(13.18) \\
\int_{0}^{\infty} e^{-2 p x} Y_{0}(a x) J_{0}(a x) x d x=-\frac{k}{2 \pi p^{2}}[K(k)-E(k)] . \\
\int_{0}^{\infty} e^{-2 p x} Y_{0}(a x) J_{0}(a x) x^{2} d x=-\frac{k}{4 \pi p^{3}}\left[\left(1+k^{2}\right) K(k)-\left(1+2 k^{2}\right) E(k)\right] . \\
\int_{0}^{\infty} e^{-2 p x} Y_{0}(a x) J_{0}(a x) x^{3} d x=-\frac{k}{8 \pi p^{4}}\left[2\left(1-2 k^{4}\right) K(k)+\left(2+k^{2}+8 k^{4}\right) E(k)\right] .
\end{gathered}
$$

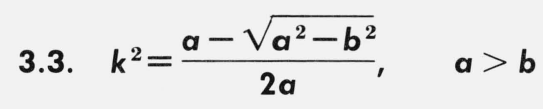

$$
\begin{gathered}
\int_{0}^{\infty} Y_{0}\left(a x^{2}\right) J_{0}\left(b x^{2}\right) d x=-\frac{\Gamma^{2}\left(\frac{1}{4}\right)}{2 \pi^{2} \sqrt{a}} K(k) . \\
\int_{0}^{\infty} Y_{0}\left(a x^{2}\right) J_{0}\left(b x^{2}\right) x^{2} d x=\frac{\Gamma^{2}\left(\frac{3}{4}\right)}{\pi^{2} \sqrt{a\left(a^{2}-b^{2}\right)}} K(k) .
\end{gathered}
$$




$$
\begin{aligned}
& \int_{0}^{\infty} Y_{0}\left(a x^{2}\right) J_{1}\left(b x^{2}\right) d x=\frac{4 \Gamma^{2}\left(\frac{3}{4}\right) \sqrt{a}}{\pi^{2} b}\left[E(k)-\left(1-k^{2}\right) K(k)\right] . \\
& \int_{0}^{\infty} Y_{0}\left(a x^{2}\right) J_{2}\left(b x^{2}\right) d x=-\frac{2 \Gamma^{2}\left(\frac{1}{4}\right) \sqrt{a^{3}}}{9 \pi^{2} b^{2}}\left[\left(2-3 k^{2}\right)\left(1-k^{2}\right) K(k)-2\left(1-2 k^{2}\right) E(k)\right] . \\
& \int_{0}^{\infty} Y_{1}\left(a x^{2}\right) J_{1}\left(b x^{2}\right) d x=-\frac{\Gamma^{2}\left(\frac{1}{4}\right) \sqrt{a}}{3 \pi^{2} b}\left[\left(1-k^{2}\right) K(k)-\left(1-2 k^{2}\right) E(k)\right] . \\
& \int_{0}^{\infty} Y_{1}\left(a x^{2}\right) J_{1}\left(b x^{2}\right) x^{2} d x=\frac{2 \Gamma^{2}\left(\frac{3}{4}\right) \sqrt{a}}{\pi^{2} b \sqrt{a^{2}-b^{2}}}\left[\left(1-k^{2}\right) K(k)-\left(1-2 k^{2}\right) \boldsymbol{E}(k)\right] . \\
& \int_{0}^{\infty} Y_{1}\left(a x^{2}\right) J_{2}\left(b x^{2}\right) d x=\frac{8 \Gamma^{2}\left(\frac{3}{4}\right) \sqrt{a^{3}}}{5 \pi^{2} b^{2}}\left[2\left(1-k^{2}+k^{4}\right) E(k)-\left(2-k^{2}\right)\left(1-k^{2}\right) K(k)\right] . \\
& \int_{0}^{\infty} Y_{2}\left(a x^{2}\right) J_{2}\left(b x^{2}\right) d x=-\frac{2 \Gamma^{2}\left(\frac{1}{4}\right) \sqrt{a^{3}}}{21 \pi^{2} b^{2}}\left[\left(2+5 k^{2}-8 k^{4}\right) \quad\left(1-k^{2}\right) K(k)\right. \\
& \left.-2\left(1-2 k^{2}\right)\left(1+4 k^{2}-4 k^{4}\right) E(k)\right] . \\
& \text { 3.4. } k^{2}=\frac{b^{2}}{a^{2}+b^{2}} \\
& \int_{0}^{\infty} Y_{0}(a x) K_{0}(b x) d x=-\frac{k}{b} K(k) .
\end{aligned}
$$

\section{Integrands Involving Modified Bessel Functions of the First Kind 4}

$$
\begin{aligned}
& \text { 4.1. } k^{2}=\frac{2 a}{p+a}, \quad p>a \\
& \int_{0}^{\infty} e^{-p x^{2}} I_{0}\left(a x^{2}\right) d x=\frac{k}{\sqrt{2 \pi a}} K(k) . \\
& \int_{0}^{\infty} e^{-p x^{2}} \boldsymbol{I}_{0}\left(a x^{2}\right) x^{2} d x=\frac{k^{3}}{4\left(1-k^{2}\right) \sqrt{2 \pi a^{3}}} E(k) . \\
& \int_{0}^{\infty} e^{-p x^{2}} I_{0}\left(a x^{2}\right) x^{4} d x=\frac{k^{5}}{16\left(1-k^{2}\right)^{2} \sqrt{2 \pi a^{5}}}\left[2\left(2-k^{2}\right) E(k)-\left(1-k^{2}\right) K(k) .\right]
\end{aligned}
$$

${ }^{4}$ The integrals of the same types as compiled in 2.1-2.6, 6.1-6.7 which involve $I_{n}(x)$ in place of $J_{n}(x)$ are also expressible in terms of $K(k)$ and $E(k)$, whose results may be deduced from those without difficulty by using the relation $J_{n}(i x)=i^{n} I_{n}(x)$. Some of those results are also found in: WA 391 (5); OL 122 (13.21)-
$(13.23), 123(13.24)-(13.27)$; OB $152(2.30),(2.31), 153(2.38), 154(2.40),(2.41)$; BY $251(562.01)-(562.04)$. 


$$
\int_{0}^{\infty} e^{-p x^{2}} I_{0}\left(a x^{2}\right) x^{6} d x=\frac{k^{7}}{64\left(1-k^{2}\right)^{3} \sqrt{2 \pi a^{7}}}\left[\left(23-23 k^{2}+8 k^{4}\right) E(k)-4\left(1-k^{2}\right)\left(2-k^{2}\right) K(k)\right] .
$$

$$
\int_{0}^{\infty} e^{-p x^{2}} I_{1}\left(a x^{2}\right) d x=\frac{1}{k \sqrt{2 \pi a}}\left[\left(2-k^{2}\right) K(k)-2 E(k)\right] .
$$

$$
\int_{0}^{\infty} e^{-p x^{2}} I_{1}\left(a x^{2}\right) x^{2} d x=\frac{k}{4\left(1-k^{2}\right) \sqrt{2 \pi a^{3}}}\left[\left(2-k^{2}\right) E(k)-2\left(1-k^{2}\right) K(k)\right] .
$$

$\int_{0}^{\infty} e^{-p x^{2}} I_{1}\left(a x^{2}\right) x^{4} d x=\frac{k^{3}}{16\left(1-k^{2}\right)^{2} \sqrt{2 \pi a^{5}}}\left[2\left(1-k^{2}+k^{4}\right) E(k)-\left(2-k^{2}\right)\left(1-k^{2}\right) K(k)\right]$.

NM 151

$$
\int_{0}^{\infty} e^{-p x^{2}} I_{1}\left(a x^{2}\right) x^{-2} d x=\frac{2 \sqrt{2 a}}{3 \sqrt{\pi k^{3}}}\left[\left(2-k^{2}\right) E(k)-2\left(1-k^{2}\right) K(k)\right] .
$$

$$
\int_{0}^{\infty} e^{-p x^{2}} I_{2}\left(a x^{2}\right) d x=\frac{1}{3 k^{3} \sqrt{2 \pi a}}\left[\left(16-16 k^{2}+3 k^{4}\right) K(k)-8\left(2-k^{2}\right)\left(1-k^{2}\right) E(k)\right] .
$$

$$
\int_{0}^{\infty} e^{-p x^{2}} I_{2}\left(a x^{2}\right) x^{2} d x=\frac{1}{4 k\left(1-k^{2}\right) \sqrt{2 \pi a^{3}}},\left[\left(16-16 k^{2}+k^{4}\right) E(k)-8\left(1-k^{2}\right)\left(2-k^{2}\right) K(k)\right] .
$$

$$
\begin{aligned}
& \int_{0}^{\infty} e^{-p x^{2}} I_{2}\left(a x^{2}\right) x^{4} d x=\frac{k}{16\left(1-k^{2}\right)^{2} \sqrt{2 \pi a^{5}}} {\left[\left(16-16 k^{2}-k^{4}\right)\right.} \\
&\left.\times\left(1-k^{2}\right) K(k)-2\left(2-k^{2}\right)\left(4-4 k^{2}-k^{4}\right) E(k)\right] .
\end{aligned}
$$

$$
\begin{aligned}
& \int_{0}^{\infty} e^{-p x^{2} I_{2}}\left(a x^{2}\right) x^{6} d x=\frac{k^{3}}{64\left(1-k^{2}\right)^{3} \sqrt{2 \pi a^{7}}}\left[4\left(2-2 k^{2}-k^{4}\right)\right. \\
& \left.\times\left(2-k^{2}\right)\left(1-k^{2}\right) K(k)-\left(16-32 k^{2}+9 k^{4}+7 k^{6}-8 k^{8}\right) E(k)\right] . \\
& \int_{0}^{\infty} e^{-p x^{2}} I_{2}\left(a x^{2}\right) x^{-2} d x=\frac{2 \sqrt{2 a}}{15 \sqrt{\pi k^{5}}}\left[\left(16-16 k^{2}+k^{4}\right) E(k)-8\left(2-k^{2}\right)\left(1-k^{2}\right) K(k)\right] .
\end{aligned}
$$

$$
\begin{aligned}
& \int_{0}^{\infty} e^{-p x^{2}} I_{3}\left(a x^{2}\right) d x=\frac{1}{15 k^{5} \sqrt{2 \pi a}}\left[\left(128-128 k^{2}+15 k^{4}\right)\right. \\
&\left.\times\left(2-k^{2}\right) K(k)-2\left(128-128 k^{2}+23 k^{4}\right) E(k)\right] .
\end{aligned}
$$

$$
\begin{aligned}
& \int_{0}^{\infty} e^{-p x^{2}} I_{3}\left(a x^{2}\right) x^{2} d x=\frac{1}{12 k^{3}\left(1-k^{2}\right)} \sqrt{2 \pi a^{3}} \\
&\left.\times\left(2-k^{2}\right) E(k)-2\left(1-k^{2}\right)\left(128-128 k^{2}+27 k^{4}\right) K(k)\right] .
\end{aligned}
$$

$$
\begin{aligned}
\int_{0}^{\infty} e^{-p x^{2}} I_{3}\left(a x^{2}\right) x^{4} d x= & \frac{1}{16 k\left(1-k^{2}\right)^{2} \sqrt{2 \pi a^{5}}}\left[\left(128-128 k^{2}-k^{4}\right)\right. \\
& \left.\times\left(2-k^{2}\right)\left(1-k^{2}\right) K(k)-2\left(128-256 k^{2}+135 k^{4}-7 k^{6}-k^{8}\right) E(k)\right] .
\end{aligned}
$$




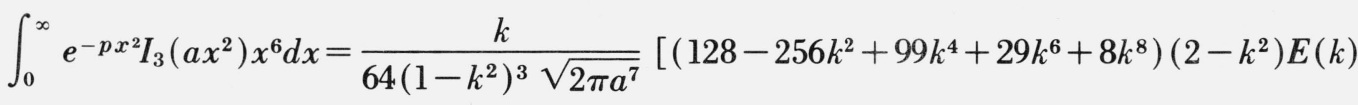

$$
\begin{aligned}
& \left.-2\left(1-k^{2}\right)\left(128-256 k^{2}+123 k^{4}+5 k^{6}+2 k^{8}\right) K(k)\right] . \\
& \int_{0}^{\infty} e^{-p x^{2}} I_{3}\left(a x^{2}\right) x^{-2} d x=\frac{2 \sqrt{2 a}}{105 \sqrt{\pi k^{7}}}\left[\left(128-128 k^{2}+3 k^{4}\right)\right. \\
& \left.\times\left(2-k^{2}\right) E(k)-2\left(1-k^{2}\right)\left(128-128 k^{2}+27 k^{4}\right) K(k)\right] . \\
& \int_{0}^{\infty} e^{-p x^{2}} I_{3}\left(a x^{2}\right) x^{-4} d x=\frac{8 \sqrt{2 a^{3}}}{945 \sqrt{\pi k^{9}}}\left[\left(128-128 k^{2}-k^{4}\right)\left(1-k^{2}\right)\right. \\
& \left.\times\left(2-k^{2}\right) K(k)-2\left(128-256 k^{2}+135 k^{4}-7 k^{6}-k^{8}\right) E(k)\right] . \\
& \int_{0}^{\infty} e^{-p x^{2}} I_{3}\left(a x^{2}\right) x^{-6} d x=\frac{32 \sqrt{2 a^{5}}}{10395 \sqrt{\pi k^{11}}}\left[\left(128-256 k^{2}+99 k^{4}+29 k^{6}+8 k^{8}\right)\right. \\
& \left.\times\left(2-k^{2}\right) E(k)-2\left(1-k^{2}\right)\left(128-256 k^{2}+123 k^{4}+5 k^{6}+2 k^{8}\right) K(k)\right] .
\end{aligned}
$$$$
4.2 \quad k^{2}=\frac{1}{b^{2}}(\sqrt{a+b}-\sqrt{a})^{2}(\sqrt{a+2 b}-\sqrt{a+b})^{2}, \quad k^{2}+k^{\prime 2}=1
$$$$
\int_{0}^{\infty} e^{-2(a+b) x} I_{0}^{2}(a x) I_{0}(2 b x) d x=\frac{4}{\pi^{2} b \sqrt{a}}(\sqrt{a+2 b}-\sqrt{a+b}) \quad K(k) K\left(k^{\prime}\right) . \quad \text { MV } 228
$$

$$
\text { 4.3. } k^{2}=\left(\frac{p-a}{p+a}\right)^{2}
$$

$$
\begin{gathered}
\int_{0}^{\infty} e^{-2 p x} I_{0}(a x) K_{0}(a x) d x=\frac{1}{p+a} K(k) . \quad \text { ET II } 370 \text { (48) } \\
\int_{0}^{\infty} e^{-2 p x} I_{0}(a x) K_{0}(a x) x d x=\frac{1}{4 p\left(a^{2}-p^{2}\right)}[(p+a) E(k)-2 p K(k)] . \\
\int_{0}^{\infty} e^{-2 p x} I_{0}(a x) K_{0}(a x) x^{2} d x=\frac{1}{8 p^{2}(a+p)(a-p)^{2}}\left[\left(a^{2}-3 p^{2}\right) E(k)+2 p(2 p-a) K(k)\right] . \\
4.4 . \quad \boldsymbol{k}^{2}=\left(\frac{\mathbf{b}}{\sqrt{\mathbf{a}^{2}+\mathbf{b}^{2}}+\mathbf{a}}\right)^{2}, \quad \boldsymbol{k}^{2}+\boldsymbol{k}^{\prime 2}=1 \\
\int_{0}^{\infty} I_{0}(a x) K_{0}(a x) J_{0}(2 b x) d x=\frac{k^{\prime 2}}{\pi b} K(k) K\left(k^{\prime}\right) . \\
\int_{0}^{\infty} I_{0}(a x) K_{0}(a x) J_{2}(2 b x) d x=\frac{1}{\pi b k^{\prime 2}}\left[2 E(k)-\left(1-k^{2}\right) K(k)\right]\left[\left(2-k^{\prime 2}\right) K\left(k^{\prime}\right)-2 E\left(k^{\prime}\right)\right] . \\
\text { OB } 23(2.116) \\
I_{1}(a x) K_{1}(a x) J_{0}(2 b x) d x=\frac{k^{\prime 2}}{\pi b k^{2}} E\left(k^{\prime}\right)[K(k)-E(k)] .
\end{gathered}
$$




$$
\begin{aligned}
& \begin{aligned}
\int_{0}^{\infty} I_{1}(a x) K_{1}(a x) J_{2}(2 b x) d x=\frac{1}{3 \pi b k^{2} k^{\prime 2}} & {\left[\left(1+k^{2}\right) E(k)\right.} \\
& \left.-\left(1-k^{2}\right) K(k)\right]\left[\left(2-k^{\prime 2}\right) E\left(k^{\prime}\right)-2\left(1-k^{\prime 2}\right) K\left(k^{\prime}\right)\right] . \\
\int_{0}^{\infty} I_{2}(a x) K_{2}(a x) J_{0}(2 b x) d x=\frac{k^{\prime 2}}{9 \pi b k^{4}}[ & \left(2+k^{2}\right) K(k) \\
- & \left.2\left(1+k^{2}\right) E(k)\right]\left[2\left(2-k^{\prime 2}\right) E\left(k^{\prime}\right)-\left(1-k^{\prime 2}\right) K\left(k^{\prime}\right)\right] .
\end{aligned}
\end{aligned}
$$

\section{Integrands Involving Modified Bessel Functions of the Second Kind}

$$
\begin{aligned}
& \text { 5.1. } k_{1}^{2}=\frac{p-a}{p+a}, \quad k_{2}^{2}=\frac{a-p}{2 a} \\
& \int_{0}^{\infty} e^{-p x^{2}} K_{0}\left(a x^{2}\right) d x=\frac{\sqrt{\pi}}{\sqrt{p+a}} K\left(k_{1}\right), \quad p>a ; \\
& =\frac{\sqrt{\pi}}{\sqrt{2 a}} K\left(k_{2}\right), \quad p<a . \\
& \int_{0}^{\infty} e^{-p x^{2}} K_{0}\left(a x^{2}\right) x^{2} d x=\frac{\sqrt{\pi(p+a)}}{2\left(p^{2}-a^{2}\right)}\left[K\left(k_{1}\right)-E\left(k_{1}\right)\right], \quad p>a ; \\
& =\frac{\sqrt{\pi}}{2\left(a^{2}-p^{2}\right) \sqrt{2 a}}\left[2 a E\left(k_{2}\right)-(p+a) K\left(k_{2}\right)\right], \quad p<a . \\
& \int_{0}^{\infty} e^{-p x^{2}} K_{0}\left(a x^{2}\right) x^{4} d x=\frac{\sqrt{\pi(p+a)}}{4\left(p^{2}-a^{2}\right)^{2}}\left[(3 p+a) K\left(k_{1}\right)-4 p E\left(k_{1}\right)\right], \quad p>a ; \\
& =\frac{\sqrt{\pi}}{4\left(a^{2}-p^{2}\right)^{2} \sqrt{2 a}}\left[(p+a)(3 p+a) K\left(k_{2}\right)-8 p a E\left(k_{2}\right)\right], \quad p<a . \\
& \int_{0}^{\infty} e^{-p x^{2}} K_{0}\left(a x^{2}\right) x^{6} d x=\frac{\sqrt{\pi(p+a)}}{8\left(p^{2}-a^{2}\right)^{3}}\left[\left(15 p^{2}+8 p a+9 a^{2}\right) K\left(k_{1}\right)-\left(23 p^{2}+9 a^{2}\right) E\left(k_{1}\right)\right], \quad p>a ; \\
& =\frac{\sqrt{\pi}}{8\left(a^{2}-p^{2}\right)^{3} \sqrt{2 a}}\left[2 a\left(23 p^{2}+9 a^{2}\right) E\left(k_{2}\right)-(p+a)\left(15 p^{2}+8 p a+9 a^{2}\right) K\left(k_{2}\right)\right], \quad p<a . \\
& \int_{0}^{\infty} e^{-p x^{2}} K_{1}\left(a x^{2}\right) x^{2} d x=\frac{\sqrt{\pi(p+a)}}{2 a\left(p^{2}-a^{2}\right)}\left[p E\left(k_{1}\right)-a K\left(k_{1}\right)\right], \quad p>a \\
& =\frac{\sqrt{\pi}}{2\left(a^{2}-p^{2}\right) \sqrt{2 a}}\left[(p+a) K\left(k_{2}\right)-2 p E\left(k_{2}\right)\right], \quad p<a . \\
& \int_{0}^{\infty} e^{-p x^{2}} K_{1}\left(a x^{2}\right) x^{4} d x=\frac{\sqrt{\pi(p+a)}}{4 a\left(p^{2}-a^{2}\right)^{2}}\left[\left(p^{2}+3 a^{2}\right) E\left(k_{1}\right)-a(p+3 a) K\left(k_{1}\right)\right], \quad p>a \\
& =\frac{\sqrt{\pi}}{4\left(a^{2}-p^{2}\right)^{2} \sqrt{2 a}}\left[2\left(p^{2}+3 a^{2}\right) E\left(k_{2}\right)-(p+a)(p+3 a) K\left(k_{2}\right)\right], \quad p<a .
\end{aligned}
$$

\footnotetext{
${ }^{5}$ For negative $p$, the second results are available.
} 


$$
\begin{aligned}
\int_{0}^{\infty} e^{-p x^{2}} K_{1}\left(a x^{2}\right) x^{6} d x & =\frac{\sqrt{\pi(p+a)}}{8 a\left(p^{2}-a^{2}\right)^{3}}\left[p\left(3 p^{2}+29 a^{2}\right) E\left(k_{1}\right)\right. \\
& =\frac{\sqrt{\pi}}{8\left(a^{2}-p^{2}\right)^{3} \sqrt{2 a}}\left[(p+a)\left(3 p^{2}+24 p a+5 a^{2}\right) K\left(k_{1}\right)\right], \quad p>a ;
\end{aligned}
$$

$$
\begin{aligned}
\int_{0}^{\infty} e^{-p x^{2}} K_{2}\left(a x^{2}\right) x^{4} d x & =\frac{\sqrt{\pi(p+a)}}{4 a^{2}\left(p^{2}-a^{2}\right)^{2}}\left[a\left(5 a^{2}+3 p a-4 p^{2}\right) K\left(k_{1}\right)\right. \\
& =\frac{\sqrt{\pi}}{4 a\left(a^{2}-p^{2}\right)^{2} \sqrt{2 a}}\left[\left(5 a^{2}+3 p a-4 p^{2}\right)\right. \\
\left.\left.\times(p+a) K\left(k_{2}\right)-8 p\left(2 a^{2}-p^{2}\right) E\left(k_{1}\right)\right], E\left(k_{2}\right)\right], & p>a ; \\
\times & \quad p<.
\end{aligned}
$$

5.2. $k_{1}^{2}=\frac{a-\sqrt{a^{2}-b^{2}}}{2 a}, \quad k_{2}^{2}=\frac{b-\sqrt{b^{2}-a^{2}}}{2 b}$

$$
k_{1}^{2}+k_{1}^{\prime 2}=1, \quad k_{2}^{2}+k_{2}^{\prime 2}=1
$$

$$
\begin{gathered}
\int_{0}^{\infty} K_{0}\left(a x^{2}\right) K_{0}\left(b x^{2}\right) d x=\frac{\Gamma^{2}\left(\frac{1}{4}\right)}{4 \sqrt{2 a}}\left[K\left(k_{1}\right)+K\left(k_{1}^{\prime}\right)\right], \quad a>b . \quad \text { OB } 153(2.33) \\
\int_{0}^{\infty} K_{0}\left(a x^{2}\right) K_{0}\left(b x^{2}\right) x^{2} d x=\frac{\Gamma^{2}\left(\frac{3}{4}\right)}{2 \sqrt{2 a^{3}}\left(k_{1}^{\prime 2}-k_{1}^{2}\right)}\left[K\left(k_{1}^{\prime}\right)-K\left(k_{1}\right)\right], \quad a>b .
\end{gathered}
$$

$$
\begin{aligned}
& \int_{0}^{\infty} K_{1}\left(a x^{2}\right) K_{0}\left(b x^{2}\right) x^{2} d x \\
& =\frac{\Gamma^{2}\left(\frac{1}{4}\right)}{8 \sqrt{2 a^{3}}\left(k_{1}^{\prime 2}-k_{1}^{2}\right)}\left[2 E\left(k_{1}\right)-K\left(k_{1}\right)+K\left(k_{1}^{\prime}\right)-2 E\left(k_{1}^{\prime}\right)\right], \quad a>b \\
& =\frac{a \Gamma^{2}\left(\frac{1}{4}\right)}{16 \sqrt{2 b^{5}}\left(k_{2}^{\prime 2}-k_{2}^{2}\right)\left(k_{2} k_{2}^{\prime}\right)^{2}}\left[\left(1-k_{2}^{2}\right) K\left(k_{2}\right)-E\left(k_{2}\right)+E\left(k_{2}^{\prime}\right)-\left(1-k_{2}^{\prime 2}\right) K\left(k_{2}^{\prime}\right)\right], \quad a<b .
\end{aligned}
$$

$$
\begin{gathered}
5.3 \boldsymbol{k}^{2}=\left(\frac{\mathbf{a}-\mathbf{b}}{\mathbf{a}+\mathbf{b}}\right)^{2} \\
\int_{0}^{\infty} K_{0}(a x) K_{0}(b x) d x=\frac{\pi}{a+b} K(k) .
\end{gathered}
$$




$$
\begin{aligned}
& \int_{0}^{\infty} K_{0}(a x) K_{0}(b x) x^{2} d x=\frac{\pi}{(a+b)(a-b)^{2}}[K(k)-E(k)] . \\
& \int_{0}^{\infty} K_{1}(a x) K_{0}(b x) x d x=\frac{\pi}{2 a\left(a^{2}-b^{2}\right)}[2 a K(k)-(a+b) E(k)] . \\
& \int_{0}^{\infty} K_{1}(a x) K_{0}(b x) x^{3} d x=\frac{\pi}{2 a(a+b)^{2}(a-b)^{3}}\left[2 a(3 a+b) K(k)-\left(7 a^{2}+b^{2}\right) E(k)\right] . \\
& \int_{0}^{\infty} K_{1}(a x) K_{1}(b x) x^{2} d x=\frac{\pi}{2 a b(a+b)(a-b)^{2}}\left[\left(a^{2}+b^{2}\right) E(k)-2 a b K(k)\right] . \\
& \int_{0}^{\infty} K_{2}(a x) K_{0}(b x) x^{2} d x=\frac{\pi}{a^{2}(a+b)(a-b)^{2}}\left[a(3 a-2 b) K(k)-\left(2 a^{2}-b^{2}\right) E(k)\right] . \\
& \int_{0}^{\infty} K_{2}(a x) K_{1}(b x) x^{3} d x=\frac{\pi}{2 a^{2} b(a+b)^{2}(a-b)^{3}}\left[\left(3 a^{4}+7 a^{2} b^{2}\right.\right. \\
& \left.\left.-2 b^{4}\right) E(k)-2 a b\left(3 a^{2}+3 a b-2 b^{2}\right) K(k)\right] . \\
& 5.4 k_{1}^{2}=\frac{a-\sqrt{a^{2}-b^{2}}}{2 a}, \quad k_{2}^{2}=\frac{b-\sqrt{b^{2}-a^{2}}}{2 b} \\
& k_{1}^{2}+k_{1}^{\prime 2}=1, \quad k_{2}^{2}+k_{2}^{\prime 2}=1 \\
& \int_{0}^{\infty} K_{0}^{2}(a x) K_{0}(2 b x) d x=\frac{\pi}{2 a} K\left(k_{1}\right) K\left(k_{1}^{\prime}\right), \quad a>b ; \\
& =\frac{\pi}{4 b}\left[K^{2}\left(k_{2}\right)+K^{2}\left(k_{2}^{\prime}\right)\right], \quad a<b .
\end{aligned}
$$

\section{Integrands Involving Products of Bessel and Modified Bessel Functions}

$$
\begin{aligned}
& 6.1 \quad k_{1}^{2}=\frac{2}{1+\sqrt{1+a^{2} \gamma^{2}}}, \quad k_{2}^{2}=\frac{1-\sqrt{1-b^{2} \gamma^{2}}}{2} \\
& \gamma^{2}=\frac{2}{p^{2}-a^{2}+b^{2}+\sqrt{\left(p^{2}-a^{2}+b^{2}\right)^{2}+4 a^{2} b^{2}}} \\
& \int_{0}^{\infty} e^{-p x^{2}} K_{0}\left(a x^{2}\right) J_{0}\left(b x^{2}\right) d x=\frac{k_{1} \sqrt{\gamma}}{\sqrt{\pi}} K\left(k_{1}\right) K\left(k_{2}\right) . \\
& \int_{0}^{\infty} e^{-p x^{2}} K_{0}\left(a x^{2}\right) J_{1}\left(b x^{2}\right) d x=\frac{\sqrt{\gamma}}{k_{1} k_{2} \sqrt{\pi\left(1-k_{2}^{2}\right)}}\left[\left(2-k_{1}^{2}\right) K\left(k_{1}\right)\right. \\
& \left.-2 E\left(k_{1}\right)\right]\left[E\left(k_{2}\right)-\left(1-k_{2}^{2}\right) K\left(k_{2}\right)\right] .
\end{aligned}
$$




$$
\begin{aligned}
& \int_{0}^{\infty} e^{-p x^{2}} K_{0}\left(a x^{2}\right) J_{2}\left(b x^{2}\right) d x=\frac{\sqrt{\gamma}}{9 \sqrt{\pi} k_{1}^{3} k_{2}^{2}\left(1-k_{2}^{2}\right)} \\
& \times\left[\left(16-16 k_{1}^{2}+3 k_{1}^{4}\right) K\left(k_{1}\right)-8\left(2-k_{1}^{2}\right) E\left(k_{1}\right)\right] \\
& \times\left[\left(1-k_{2}^{2}\right)\left(2-3 k_{2}^{2}\right) K\left(k_{2}\right)-2\left(1-2 k_{2}^{2}\right) E\left(k_{2}\right)\right] . \\
& \int_{0}^{\infty} e^{-p x^{2}} K_{1}\left(a x^{2}\right) J_{1}\left(b x^{2}\right) d x=\frac{\sqrt{\gamma}}{3 k_{1} k_{2} \sqrt{\pi\left(1-k_{1}^{2}\right)\left(1-k_{2}^{2}\right)}} \\
& \times\left[\left(2-k_{1}^{2}\right) E\left(k_{1}\right)-2\left(1-k_{1}^{2}\right) K\left(k_{1}\right)\right]\left[\left(1-k_{2}^{2}\right) K\left(k_{2}\right)-\left(1-2 k_{2}^{2}\right) E\left(k_{2}\right)\right] . \\
& \int_{0}^{\infty} e^{-p x^{2}} K_{1}\left(a x^{2}\right) J_{2}\left(b x^{2}\right) d x=\frac{\sqrt{\gamma}}{15 \sqrt{\pi} k_{1}^{3} k_{2}^{2}\left(1-k_{2}^{2}\right) \sqrt{1-k_{1}^{2}}} \\
& \times\left[\left(16-16 k_{1}^{2}+k_{1}^{4}\right) E\left(k_{1}\right)-8\left(1-k_{1}^{2}\right)\left(2-k_{1}^{2}\right) K\left(k_{1}\right)\right] \\
& \times\left[2\left(1-k_{2}^{2}+k_{2}^{4}\right) \boldsymbol{E}\left(k_{2}\right)-\left(1-k_{2}^{2}\right)\left(2-k_{2}^{2}\right) K\left(k_{2}\right)\right] . \\
& \int_{0}^{\infty} e^{-p x^{2}} K_{2}\left(a x^{2}\right) J_{2}\left(b x^{2}\right) d x=\frac{\sqrt{\gamma}}{105 \sqrt{\pi} k_{1}^{3} k_{2}^{2}\left(1-k_{1}^{2}\right)\left(1-k_{2}^{2}\right)} \\
& \times\left[\left(1-k_{1}^{2}\right)\left(16-16 k_{1}^{2}-k_{1}^{4}\right) K\left(k_{1}\right)-2\left(2-k_{1}^{2}\right)\left(4-4 k_{1}^{2}-k_{1}^{4}\right) E\left(k_{1}\right)\right] \\
& \times\left[\left(1-k_{2}^{2}\right)\left(2+5 k_{2}^{2}-8 k_{2}^{4}\right) K\left(k_{2}\right)-2\left(1-2 k_{2}^{2}\right)\left(1+4 k_{2}^{2}-4 k_{2}^{4}\right) E\left(k_{2}\right)\right] . \\
& \text { 6.2. } k^{2}=\left(\frac{b}{\sqrt{a^{2}+b^{2}}+a}\right)^{2} \\
& \int_{0}^{\infty} K_{0}\left(a x^{2}\right) J_{0}\left(b x^{2}\right) d x=\frac{\Gamma^{2}\left(\frac{1}{4}\right) \sqrt{k}}{2 \pi \sqrt{b}} K(k) . \\
& \int_{0}^{\infty} K_{0}\left(a x^{2}\right) J_{0}\left(b x^{2}\right) x^{2} d x=\frac{\Gamma^{2}\left(\frac{3}{4}\right) \sqrt{k}}{\pi \sqrt{b\left(a^{2}+b^{2}\right)}} K(k) . \quad \text { OB } 21(2.107) \\
& \int_{0}^{\infty} K_{0}\left(a x^{2}\right) J_{1}\left(b x^{2}\right) d x=\frac{2 \Gamma^{2}\left(\frac{3}{4}\right)}{\pi \sqrt{b k}}[K(k)-E(k)] . \\
& \int_{0}^{\infty} K_{0}\left(a x^{2}\right) J_{2}\left(b x^{2}\right) d x=\frac{\Gamma^{2}\left(\frac{1}{4}\right)}{18 \pi \sqrt{b k^{3}}}\left[\left(2+k^{2}\right) K(k)-2\left(1+k^{2}\right) E(k)\right] . \\
& \int_{0}^{\infty} K_{1}\left(a x^{2}\right) J_{1}\left(b x^{2}\right) d x=\frac{\Gamma^{2}\left(\frac{1}{4}\right) \sqrt{b}}{12 \pi a \sqrt{k^{3}}}\left[\left(1+k^{2}\right) E(k)-\left(1-k^{2}\right) K(k)\right] . \\
& \int_{0}^{\infty} K_{1}\left(a x^{2}\right) J_{1}\left(b x^{2}\right) x^{2} d x=\frac{\Gamma^{2}\left(\frac{3}{4}\right) \sqrt{b}}{2 \pi a \sqrt{\left(a^{2}+b^{2}\right) k^{3}}}\left[\left(1+k^{2}\right) E(k)-\left(1-k^{2}\right) K(k)\right] .
\end{aligned}
$$




$$
\begin{aligned}
& \int_{0}^{\infty} K_{1}\left(a x^{2}\right) J_{2}\left(b x^{2}\right) d x=\frac{\Gamma^{2}\left(\frac{3}{4}\right) \sqrt{b}}{5 \pi a \sqrt{k^{5}}}\left[2\left(1-k^{2}+k^{4}\right) E(k)-\left(1-k^{2}\right)\left(2-k^{2}\right) K(k)\right] . \\
& \int_{0}^{\infty} K_{2}\left(a x^{2}\right) J_{2}\left(b x^{2}\right) d x=\frac{\Gamma^{2}\left(\frac{1}{4}\right) \sqrt{b^{3}}}{168 \pi a^{2} \sqrt{k^{7}}}\left[\left(2-9 k^{2}-k^{4}\right)\right. \\
& \left.\times\left(1-k^{2}\right) K(k)-2\left(1+k^{2}\right)\left(1-6 k^{2}+k^{4}\right) E(k)\right] . \\
& \text { 6.3. } k^{2}=\frac{b^{2}}{a^{2}+b^{2}} \\
& \int_{0}^{\infty} K_{0}(a x) J_{0}(b x) d x=\frac{k}{b} K(k) . \quad \text { OB } 22(2.109), 152(2.28) \\
& \int_{0}^{\infty} K_{0}(a x) J_{0}(b x) x^{2} d x=\frac{k^{3}}{b^{3}}[2 E(k)-K(k)] . \\
& \int_{0}^{\infty} K_{0}(a x) J_{0}(b x) x^{4} d x=\frac{3 k^{5}}{b^{5}}\left[8\left(1-2 k^{2}\right) E(k)-\left(5-8 k^{2}\right) K(k)\right] . \\
& \int_{0}^{\infty} K_{0}(a x) J_{0}(b x) x^{6} d x=\frac{15 k^{7}}{b^{7}}\left[2\left(23-128 k^{2}+128 k^{4}\right) E(k)-\left(31-144 k^{2}+128 k^{4}\right) K(k)\right] . \\
& \int_{0}^{\infty} K_{0}(a x) J_{1}(b x) x d x=\frac{k}{b^{2}}[K(k)-E(k)] \\
& \int_{0}^{\infty} K_{0}(a x) J_{1}(b x) x^{3} d x=\frac{k^{3}}{b^{4}}\left[\left(1-4 k^{2}\right) K(k)-\left(1-8 k^{2}\right) E(k)\right] . \\
& \int_{0}^{\infty} K_{0}(a x) J_{1}(b x) x^{5} d x=\frac{3 k^{5}}{b^{6}}\left[\left(3-4 k^{2}\right)\left(1-16 k^{2}\right) K(k)-\left(3-88 k^{2}+128 k^{4}\right) E(k)\right] . \\
& \int_{0}^{\infty} K_{0}(a x) J_{1}(b x) x^{-1} d x=\frac{1}{k}[K(k)-E(k)] . \\
& \int_{0}^{\infty} K_{0}(a x) J_{2}(b x) d x=\frac{1}{b k}\left[\left(2-k^{2}\right) K(k)-2 E(k)\right] . \\
& \int_{0}^{\infty} K_{0}(a x) J_{2}(b x) x^{2} d x=\frac{k}{b^{3}}\left[\left(2+k^{2}\right) K(k)-2\left(1+k^{2}\right) E(k)\right] . \\
& \int_{0}^{\infty} K_{0}(a x) J_{2}(b x) x^{4} d x=\frac{k^{3}}{b^{5}}\left[\left(2+7 k^{2}-24 k^{4}\right) K(k)-2\left(1+4 k^{2}-24 k^{4}\right) E(k)\right] . \\
& \int_{0}^{\infty} K_{0}(a x) J_{2}(b x) x^{-2} d x=\frac{b}{9 k^{3}}\left[\left(2+k^{2}\right) K(k)-2\left(1+k^{2}\right) E(k)\right] . \\
& \int_{0}^{\infty} K_{0}(a x) J_{3}(b x) x d x=\frac{1}{b^{2} k}\left[\left(8-5 k^{2}\right) K(k)-\left(8-k^{2}\right) E(k)\right] . \\
& \int_{0}^{\infty} K_{0}(a x) J_{3}(b x) x^{3} d x=\frac{k}{b^{4}}\left[\left(8+3 k^{2}+4 k^{4}\right) K(k)-\left(8+7 k^{2}+8 k^{4}\right) E(k)\right] .
\end{aligned}
$$




$$
\int_{0}^{\infty} K_{0}(a x) J_{3}(b x) x^{5} d x=\frac{k^{3}}{b^{6}}\left[\left(8+19 k^{2}+60 k^{4}-192 k^{6}\right) K(k)-\left(8+23 k^{2}+72 k^{4}-384 k^{6}\right) E(k)\right] .
$$

$$
\begin{gathered}
\int_{0}^{\infty} K_{0}(a x) J_{3}(b x) x^{-1} d x=\frac{1}{9 k^{3}}\left[\left(8-5 k^{2}\right) K(k)-\left(8-k^{2}\right) E(k)\right] . \\
\int_{0}^{\infty} K_{0}(a x) J_{3}(b x) x^{-3} d x=\frac{b^{2}}{225 k^{5}}\left[\left(8+3 k^{2}+4 k^{4}\right) K(k)-\left(8+7 k^{2}+8 k^{4}\right) E(k)\right] . \\
\int_{0}^{\infty} K_{1}(a x) J_{0}(b x) x d x=\frac{k}{a b} E(k) . \\
\int_{0}^{\infty} K_{1}(a x) J_{0}(b x) x^{3} d x=\frac{k^{3}}{a b^{3}}\left[\left(7-8 k^{2}\right) E(k)-4\left(1-k^{2}\right) K(k)\right] . \\
\int_{0}^{\infty} K_{1}(a x) J_{0}(b x) x^{5} d x=\frac{3 k^{5}}{a b^{5}}\left[\left(43-168 k^{2}+128 k^{4}\right) E(k)-4\left(1-k^{2}\right)\left(7-16 k^{2}\right) K(k)\right] . \\
K_{1}(a x) J_{0}(b x) x^{7} d x=\frac{15 k^{7}}{a b^{7}}\left[\left(337-2784 k^{2}+5504 k^{4}-3072 k^{6}\right) E(k)\right. \\
\int_{0}^{\infty} K_{1}(a x) J_{1}(b x) x^{4} d x=\frac{3 k^{3}}{a b^{4}}\left[\left(1-8 k^{2}\right)\left(1-k^{2}\right) K(k)-\left(1-16 k^{2}+16 k^{4}\right) E(k)\right] . \\
\int_{0}^{\infty} K_{1}(a x) J_{1}(b x) x^{2} d x=\frac{k}{a b^{2}}\left[\left(1-k^{2}\right) K(k)-\left(1-29-176 k^{2}+192 k^{4}\right) K(k)\right] . \\
\int_{1}^{2}(b x) d x=\frac{1}{a k}\left[E(k)-\left(1-k^{2}\right) K(k)\right] .
\end{gathered}
$$$$
\int_{0}^{\infty} K_{1}(a x) J_{1}(b x) x^{6} d x=\frac{15 k^{5}}{a b^{6}}\left[\left(1-k^{2}\right)\left(3-80 k^{2}+128 k^{4}\right) K(k)-\left(3-134 k^{2}+384 k^{4}-256 k^{6}\right) E(k)\right] .
$$$$
\int_{0}^{\infty} K_{1}(a x) J_{2}(b x) x d x=\frac{1}{a b k}\left[\left(2-k^{2}\right) E(k)-2\left(1-k^{2}\right) K(k)\right] .
$$$$
\int_{0}^{\infty} K_{1}(a x) J_{2}(b x) x^{3} d x=\frac{k}{a b^{3}}\left[2\left(1-k^{2}\right)\left(1+2 k^{2}\right) K(k)-\left(2+3 k^{2}-8 k^{4}\right) E(k)\right] .
$$$$
\int_{0}^{\infty} K_{1}(a x) J_{2}(b x) x^{5} d x=\frac{3 k^{3}}{a b^{5}}\left[2\left(1-k^{2}\right)\left(1+6 k^{2}-32 k^{4}\right) K(k)-\left(2+11 k^{2}-136 k^{4}+128 k^{6}\right) E(k)\right] .
$$$$
\int_{0}^{\infty} K_{1}(a x) J_{2}(b x) x^{-1} d x=\frac{b}{3 a k^{3}}\left[\left(2-k^{2}\right) E(k)-2\left(1-k^{2}\right) K(k)\right] .
$$$$
\int_{0}^{\infty} K_{1}(a x) J_{3}(b x) d x=\frac{1}{3 a k^{3}}\left[\left(8-7 k^{2}\right) E(k)-\left(8-3 k^{2}\right)\left(1-k^{2}\right) K(k)\right] .
$$$$
\int_{0}^{\infty} K_{1}(a x) J_{3}(b x) x^{2} d x=\frac{1}{a b^{2} k}\left[\left(8-3 k^{2}-2 k^{4}\right) E(k)-\left(1-k^{2}\right)\left(8+k^{2}\right) K(k)\right] .
$$

$\int_{0}^{\infty} K_{1}(a x) J_{3}(b x) x^{4} d x=\frac{k}{a b^{4}}\left[\left(1-k^{2}\right)\left(8+13 k^{2}+24 k^{4}\right) K(k)-\left(8+9 k^{2}+16 k^{4}-48 k^{6}\right) E(k)\right]$. 


$$
\begin{aligned}
& \int_{0}^{\infty} K_{1}(a x) J_{3}(b x) x^{-2} d x=\frac{b^{2}}{45 a k^{5}}\left[\left(8-3 k^{2}-2 k^{4}\right) E(k)-\left(1-k^{2}\right)\left(8+k^{2}\right) K(k)\right] . \\
& \int_{0}^{\infty} K_{2}(a x) J_{0}(b x) x^{2} d x=\frac{k}{a^{2} b}\left[2\left(2-k^{2}\right) E(k)-\left(1-k^{2}\right) K(k)\right] . \\
& \int_{0}^{\infty} K_{2}(a x) J_{0}(b x) x^{4} d x=\frac{k^{3}}{a^{2} b^{3}}\left[2\left(19-44 k^{2}+24 k^{4}\right) E(k)-\left(1-k^{2}\right)\left(23-24 k^{2}\right) K(k)\right] . \\
& \int_{0}^{\infty} K_{2}(a x) J_{0}(b x) x^{6} d x=\frac{3 k^{5}}{a^{2} b^{5}}\left[2\left(158-923 k^{2}+1408 k^{4}-640 k^{6}\right)\right. \\
& \left.\times E(k)-\left(1-k^{2}\right)\left(211-848 k^{2}+640 k^{4}\right) K(k)\right] . \\
& \int_{0}^{\infty} K_{2}(a x) J_{1}(b x) x d x=\frac{1}{a^{2} k}\left[\left(1+k^{2}\right) E(k)-\left(1-k^{2}\right) K(k)\right] \\
& \int_{0}^{\omega} K_{2}(a x) J_{1}(b x) x^{3} d x=\frac{k}{a^{2} b^{2}}\left[\left(1-k^{2}\right)\left(3-4 k^{2}\right) K(k)-\left(3-13 k^{2}+8 k^{4}\right) E(k)\right] . \\
& \int_{0}^{\infty} K_{2}(a x) J_{1}(b x) x^{5} d x=\frac{3 k^{3}}{a^{2} b^{4}}\left[\left(1-k^{2}\right)\left(5-68 k^{2}+64 k^{4}\right) K(k)-\left(5-123 k^{2}+248 k^{4}-128 k^{6}\right) E(k)\right] . \\
& \int_{0}^{\infty} K_{2}(a x) J_{2}(b x) d x=\frac{b}{3 a^{2} k^{3}}\left[\left(1-k^{2}\right)\left(2-3 k^{2}\right) K(k)-2\left(1-2 k^{2}\right) E(k)\right] . \\
& \int_{0}^{\infty} K_{2}(a x) J_{2}(b x) x^{2} d x=\frac{1}{a^{2} b k}\left[2\left(1-k^{2}+k^{4}\right) E(k)-\left(1-k^{2}\right)\left(2-k^{2}\right) K(k)\right] . \\
& \int_{0}^{\infty} K_{2}(a x) J_{2}(b x) x^{4} d x=\frac{3 k}{a^{2} b^{3}}\left[\left(1-k^{2}\right)\left(2+5 k^{2}-8 k^{4}\right) K(k)-2\left(1-2 k^{2}\right)\left(1+4 k^{2}-4 k^{4}\right) E(k)\right] . \\
& \int_{0}^{\infty} K_{2}(a x) J_{2}(b x) x^{6} d x=\frac{15 k^{3}}{a^{2} b^{5}}\left[\left(2+15 k^{2}-144 k^{4}+128 k^{6}\right)\left(1-k^{2}\right) K(k)\right. \\
& \left.-2\left(1+7 k^{2}-135 k^{4}+256 k^{6}-128 k^{8}\right) E(k)\right] . \\
& \int_{0}^{\infty} K_{2}(a x) J_{3}(b x) x d x=\frac{1}{3 a^{2} k^{3}}\left[\left(1-k^{2}\right)\left(8-9 k^{2}\right) K(k)-\left(8-13 k^{2}+3 k^{4}\right) E(k)\right] .
\end{aligned}
$$$$
\int_{0}^{\infty} K_{2}(a x) J_{3}(b x) x^{3} d x=\frac{1}{a^{2} b^{2} k}\left[\left(1+k^{2}\right)\left(8-13 k^{2}+8 k^{4}\right) E(k)-\left(1-k^{2}\right)\left(8-k^{2}-4 k^{4}\right) K(k)\right] .
$$$$
\int_{0}^{\infty} K_{2}(a x) J_{3}(b x) x^{-1} d x=\frac{b^{2}}{15 a^{2} k^{5}}\left[\left(1-k^{2}\right)\left(8-9 k^{2}\right) K(k)-\left(8-13 k^{2}+3 k^{4}\right) E(k)\right] .
$$$$
\int_{0}^{\infty} K_{3}(a x) J_{0}(b x) x^{3} d x=\frac{k}{a^{3} b}\left[\left(23-23 k^{2}+8 k^{4}\right) E(k)-4\left(2-k^{2}\right)\left(1-k^{2}\right) K(k)\right] .
$$

$\int_{0}^{\infty} K_{3}(a x) J_{0}(b x) x^{5} d x=\frac{k^{3}}{a^{3} b^{3}}\left[\left(281-985 k^{2}+1080 k^{4}-384 k^{6}\right) E(k)\right.$

$$
\left.-4\left(1-k^{2}\right)\left(44-93 k^{2}+48 k^{4}\right) K(k)\right] .
$$




$$
\begin{aligned}
& \int_{0}^{\infty} K_{3}(a x) J_{1}(b x) x^{2} d x=\frac{1}{a^{3} k}\left[\left(3+7 k^{2}-2 k^{4}\right) E(k)-\left(1-k^{2}\right)\left(3+k^{2}\right) K(k)\right] . \\
& \int_{0}^{\infty} K_{3}(a x) J_{1}(b x) x^{4} d x=\frac{k}{a^{3} b^{2}}\left[\left(15-43 k^{2}+24 k^{4}\right)\left(1-k^{2}\right) K(k)\right. \\
& \left.-\left(15-103 k^{2}+128 k^{4}-48 k^{6}\right) E(k)\right] . \\
& \int_{0}^{\infty} K_{3}(a x) J_{2}(b x) x d x=\frac{b}{3 a^{3} k^{3}}\left[2\left(1-k^{2}\right)\left(1-3 k^{2}\right) K(k)-\left(2-7 k^{2}-3 k^{4}\right) E(k)\right] . \\
& \int_{0}^{\infty} K_{3}(a x) J_{2}(b x) x^{3} d x=\frac{1}{a^{3} b k}\left[\left(6-9 k^{2}+19 k^{4}-8 k^{6}\right) E(k)-2\left(1-k^{2}\right)\left(3-3 k^{2}+2 k^{4}\right) K(k)\right] .(52) \\
& \int_{0}^{\infty} K_{3}(a x) J_{3}(b x) d x=\frac{b^{2}}{15 a^{3} k^{5}}\left[\left(8-23 k^{2}+23 k^{4}\right) E(k)-\left(1-k^{2}\right)\left(8-19 k^{2}+15 k^{4}\right) K(k)\right] . \\
& \int_{0}^{\infty} K_{3}(a x) J_{3}(b x) x^{2} d x=\frac{1}{3 a^{3} k^{3}}\left[\left(8-15 k^{2}+3 k^{4}\right)\left(1-k^{2}\right) K(k)-\left(8-19 k^{2}+9 k^{4}-6 k^{6}\right) E(k)\right] . \\
& \int_{0}^{\infty} K_{4}(a x) J_{0}(b x) x^{4} d x=\frac{k}{a^{4} b}\left[8\left(11-11 k^{2}+6 k^{4}\right)\right. \\
& \left.\times\left(2-k^{2}\right) E(k)-\left(1-k^{2}\right)\left(71-71 k^{2}+24 k^{4}\right) K(k)\right] . \\
& \int_{0}^{\infty} K_{4}(a x) J_{1}(b x) x^{3} d x=\frac{1}{a^{4} k}\left[\left(15+58 k^{2}-33 k^{4}+8 k^{6}\right) E(k)-\left(1-k^{2}\right)\left(15+13 k^{2}-4 k^{4}\right) K(k)\right] . \\
& \int_{0}^{\infty} K_{4}(a x) J_{2}(b x) x^{2} d x=\frac{b}{a^{4} k^{3}}\left[\left(1-k^{2}\right)\left(2-9 k^{2}-k^{4}\right) K(k)-2\left(1+k^{2}\right)\left(1-6 k^{2}+k^{4}\right) E(k)\right] . \\
& \int_{0}^{\infty} K_{4}(a x) J_{3}(b x) x d x=\frac{b^{2}}{15 a^{4} k^{5}}\left[\left(8-33 k^{2}+58 k^{4}+15 k^{6}\right)\right. \\
& \left.\times E(k)-\left(1-k^{2}\right)\left(8-29 k^{2}+45 k^{4}\right) K(k)\right] . \\
& \text { 6.4. } k^{2}=\left(\frac{b}{\sqrt{a^{2}+b^{2}}+a}\right)^{2} \\
& \begin{array}{c}
\int_{0}^{\infty} K_{0}(2 a x) J_{0}^{2}(b x) d x=\frac{2 k}{\pi b}[K(k)]^{2} . \\
\int_{0}^{\infty} K_{0}(2 a x) J_{1}^{2}(b x) d x=\frac{2}{\pi b k}[K(k)-E(k)]^{2} .
\end{array} \\
& \int_{0}^{\infty} K_{0}(2 a x) J_{2}^{2}(b x) d x=\frac{2}{9 \pi b k^{3}}\left[\left(2+k^{2}\right) K(k)-2\left(1+k^{2}\right) E(k)\right]^{2} . \\
& \int_{0}^{\infty} K_{0}(2 a x) J_{3}^{2}(b x) d x=\frac{2}{225 \pi b k^{5}}\left[\left(8+3 k^{2}+4 k^{4}\right) K(k)-\left(8+7 k^{2}+8 k^{4}\right) E(k)\right]^{2} . \\
& \int_{0}^{\infty} K_{1}(2 a x) J_{0}^{2}(b x) x d x=\frac{1}{2 \pi a \sqrt{a^{2}+b^{2}}} K(k)\left[2 E(k)-\left(1-k^{2}\right) K(k)\right] . \\
& \text { OB } 153(2.37) \\
& \int_{0}^{\infty} K_{1}(2 a x) J_{1}^{2}(b x) x d x=\frac{1}{2 \pi k^{2} a \sqrt{a^{2}+b^{2}}}[K(k)-E(k)]\left[\left(1+k^{2}\right) E(k)-\left(1-k^{2}\right) K(k)\right] .
\end{aligned}
$$




$$
\begin{gathered}
\int_{0}^{\infty} K_{1}(2 a x) J_{2}^{2}(b x) x d x=\frac{1}{6 \pi k^{4} a \sqrt{a^{2}+b^{2}}}\left[2\left(1-k^{2}+k^{4}\right) E(k)-\left(1-k^{2}\right)\left(2-k^{2}\right) K(k)\right] \\
\times\left[\left(2+k^{2}\right) K(k)-2\left(1+k^{2}\right) E(k)\right] . \\
\int_{0}^{\infty} K_{2}(2 a x) J_{1}^{2}(b x) d x=\frac{b}{6 \pi a^{2} k^{3}}\left[\left(1+k^{2}\right) E(k)-\left(1-k^{2}\right) K(k)\right]^{2} . \\
\int_{0}^{\infty} K_{2}(2 a x) J_{2}^{2}(b x) d x=\frac{b}{30 \pi a^{2} k^{5}}\left[2\left(1-k^{2}+k^{4}\right) E(k)-\left(1-k^{2}\right)\left(2-k^{2}\right) K(k)\right]^{2} . \\
\int_{0}^{\infty} K_{2}(2 a x) J_{3}^{2}(b x) d x=\frac{b}{630 \pi a^{2} k^{7}}\left[\left(8-13 k^{2}+8 k^{4}\right)\left(1+k^{2}\right) E(k)\right. \\
\int_{0}^{\infty} K_{3}(2 a x) J_{1}^{2}(b x) x d x=\frac{b^{2}}{24 \pi k^{4} a^{3} \sqrt{a^{2}+b^{2}}}\left[\left(1+14 k^{2}+k^{4}\right) E(k)\right. \\
\left.\left.\left.-\left(1-k^{2}\right)\left(1+7 k^{2}\right) K(k)\right]\left[\left(1+k^{2}\right) E(k)-\left(1-k^{2}\right) K(k)\right] .4 k^{4}\right) K(k)\right]^{2}
\end{gathered}
$$

$$
\begin{gathered}
\text { 6.5. } \boldsymbol{k}^{2}=\left(\frac{\mathbf{b}}{\sqrt{\mathbf{a}^{2}+\mathbf{b}^{2}}+\mathbf{a}}\right)^{2} \\
\int_{0}^{\infty} K_{0}^{2}(a x) J_{0}(2 b x) d x=\frac{2 k}{b}[K(k)]^{2} . \quad \text { OB } 23(2.117) \\
\int_{0}^{\infty} K_{0}^{2}(a x) J_{1}(2 b x) x d x=\frac{1}{b \sqrt{a^{2}+b^{2}}} K(k)[K(k)-E(k)] . \\
\int_{0}^{\infty} K_{0}^{2}(a x) J_{2}(2 b x) d x=\frac{2}{b k}[K(k)-E(k)]^{2} . \\
\int_{0}^{\infty}(a x) J_{3}(2 b x) x d x=\frac{1}{b k^{2} \sqrt{a^{2}+b^{2}}}[K(k)-E(k)]\left[\left(2+k^{2}\right) K(k)-2\left(1+k^{2}\right) E(k)\right] . \\
\int_{0}^{\infty}(a x) J_{4}(2 b x) d x=\frac{2}{9 b k^{3}}\left[\left(2+k^{2}\right) K(k)-2\left(1+k^{2}\right) E(k)\right]^{2} .
\end{gathered}
$$

$\int_{0}^{\infty} K_{1}^{2}(a x) J_{1}(2 b x) x d x=\frac{b}{4 k^{2} a^{2} \sqrt{a^{2}+b^{2}}}$

$$
\times\left[\left(1+k^{2}\right) E(k)-\left(1-k^{2}\right) K(k)\right]\left[2 E(k)-\left(1-k^{2}\right) K(k)\right] .
$$

$$
\int_{0}^{\infty} K_{1}^{2}(a x) J_{2}(2 b x) d x=\frac{b}{6 k^{3} a^{2}}\left[\left(1+k^{2}\right) E(k)-\left(1-k^{2}\right) K(k)\right]^{2} .
$$




$$
\begin{aligned}
& 6.6 k^{2}=\left(\frac{b}{\sqrt{a^{2}+b^{2}}+a}\right)^{2} \\
& \int_{0}^{\infty} K_{0}(2 a x) J_{1}(b x) J_{0}(b x) x d x=\frac{\sqrt{a^{2}+b^{2}}}{\pi a^{2} b} K(k)[K(k)-E(k)] . \\
& \int_{0}^{\infty} K_{0}(2 a x) J_{2}(b x) J_{1}(b x) x d x=\frac{\sqrt{a^{2}+b^{2}}}{\pi a^{2} b k^{2}}[K(k)-E(k)]\left[\left(2+k^{2}\right) K(k)-2\left(1+k^{2}\right) E(k)\right] . \\
& \int_{0}^{\infty} K_{0}(2 a x) J_{3}(b x) J_{2}(b x) x d x=\frac{\sqrt{a^{2}+b^{2}}}{9 \pi a^{2} b k^{4}} \\
& \times\left[\left(2+k^{2}\right) K(k)-2\left(1+k^{2}\right) E(k)\right]\left[\left(8+3 k^{2}+4 k^{4}\right) K(k)-\left(8+7 k^{2}+8 k^{4}\right) E(k)\right] . \\
& \int_{0}^{\infty} K_{2}(2 a x) J_{1}(b x) J_{0}(b x) x d x=\frac{b \sqrt{a^{2}+b^{2}}}{4 \pi a^{4} k^{2}}\left[\left(1+k^{2}\right) E(k)-\left(1-k^{2}\right) K(k)\right]\left[2 E(k)-\left(1-k^{2}\right) K(k)\right] . \\
& \int_{0}^{\infty} K_{2}(2 a x) J_{2}(b x) J_{1}(b x) x d x=\frac{b \sqrt{a^{2}+b^{2}}}{12 \pi a^{4} k^{4}} \\
& \times\left[\left(1+k^{2}\right) E(k)-\left(1-k^{2}\right) K(k)\right]\left[2\left(1-k^{2}+k^{4}\right) E(k)-\left(1-k^{2}\right)\left(2-k^{2}\right) K(k)\right] . \\
& \int_{0}^{\infty} K_{2}(2 a x) J_{3}(b x) J_{2}(b x) x d x=\frac{b \sqrt{a^{2}+b^{2}}}{60 \pi a^{4} k^{6}}\left[2\left(1-k^{2}+k^{4}\right) E(k)-\left(1-k^{2}\right)\left(2-k^{2}\right) K(k)\right] \\
& \times\left[\left(1+k^{2}\right)\left(8-13 k^{2}+8 k^{4}\right) E(k)-\left(1-k^{2}\right)\left(8-k^{2}-4 k^{4}\right) K(k)\right] . \\
& \text { 6.7. } k^{2}=\left(\frac{b}{\sqrt{a^{2}+b^{2}}+a}\right)^{2} \\
& \int_{0}^{\infty} K_{1}(a x) K_{0}(a x) J_{0}(2 b x) x d x=\frac{1}{2 a \sqrt{a^{2}+b^{2}}} K(k)\left[2 E(k)-\left(1-k^{2}\right) K(k)\right] . \\
& \int_{0}^{\infty} K_{1}(a x) K_{0}(a x) J_{2}(2 b x) x d x=\frac{1}{2 k^{2} a \sqrt{a^{2}+b^{2}}}[K(k)-E(k)]\left[\left(1+k^{2}\right) E(k)-\left(1-k^{2}\right) K(k)\right] . \\
& \int_{0}^{\infty} K_{1}(a x) K_{0}(a x) J_{4}(2 b x) x d x=\frac{1}{6 k^{4} a \sqrt{a^{2}+b^{2}}}\left[\left(2+k^{2}\right) K(k)-2\left(1+k^{2}\right) E(k)\right] \\
& \times\left[2\left(1-k^{2}+k^{4}\right) E(k)-\left(1-k^{2}\right)\left(2-k^{2}\right) K(k)\right] . \\
& \int_{0}^{\infty} K_{2}(a x) K_{1}(a x) J_{2}(2 b x) x d x=\frac{b^{2}}{24 k^{4} a^{3} \sqrt{a^{2}+b^{2}}}\left[\left(1+k^{2}\right) E(k)-\left(1-k^{2}\right) K(k)\right] \\
& \times\left[\left(1+14 k^{2}+k^{4}\right) E(k)-\left(1-k^{2}\right)\left(1+7 k^{2}\right) K(k)\right] . \\
& \int_{0}^{\infty} K_{3}(a x) K_{2}(a x) J_{4}(2 b x) x d x=\frac{b^{4}}{3360 k^{8} a^{5} \sqrt{a^{2}+b^{2}}}\left[\left(1-k^{2}\right)\left(2-9 k^{2}-k^{4}\right) K(k)\right. \\
& \left.-2\left(1+k^{2}\right)\left(1-6 k^{2}+k^{4}\right) E(k)\right]\left[\left(1-k^{2}\right)\left(2-21 k^{2}-108 k^{4}-k^{6}\right) K(k)\right. \\
& \left.-2\left(1-11 k^{2}-108 k^{4}-11 k^{6}+k^{8}\right) E(k)\right] \text {. }
\end{aligned}
$$




$$
\begin{gathered}
\text { 6.8. } \boldsymbol{k}^{2}=\left(\frac{\mathbf{a}^{2}-b^{2}}{\mathbf{a}^{2}+b^{2}}\right)^{2} \\
\qquad \int_{0}^{\infty} K_{0}(a x) K_{0}(b x) J_{0}(a x) J_{0}(b x) x d x=\frac{1}{2\left(a^{2}+b^{2}\right)} K(k) .
\end{gathered}
$$

The author wishes to express his hearty gratitude to the late Dr. Minoru Nakagami for his invaluable advice and encouragement. His thanks are also due to Mr. Yukihiko Miyawaki and Mr. Masayuki Sawada who gave him generous assistance in carrying out this work.

\section{References}

For preparation of this paper the author referred to general expressions of these types of integrals in terms of hypergeometric functions or Legendre functions. The section numbers and the general expressions referred there are:

2.1 WA 385 (2)(3),ET II 29 (6); 2.2 deduced from ET I 196 (12); 2.3,2.4 LU 319 (28); 2.5,2.6 WA 389 (1)(2), LU 319 (26)(27);

2.7 WA 401 (2),407 (1); 2.8 ET II 52 (33); 3.1 WA 385 (4),ET II 105 (2); 3.3 ET I 332 (37); 4.1 GW 199 (6a),OL 149 (15.8);

5.1 ET II 131 (23); 5.2,5.3 ET II 145 (49); 6.1 deduced from ET I 198 (30); 6.2,6.3 WA 410 (1); 6.4 ET I 138 (18)(19); 6.5

ET II 66 (27)(28); 6.6 ET II 138 (20); 6.7 ET II 67 (29); 6.8 ET II 373 (10).

\section{References Cited in This Paper}

BY Byrd, P. F., and Friedman, M. D., Handbook of Elliptic Integrals for Engineers and Scientists, second edition, revised (Springer-Verlag, New York 1971).

ET I Erdélyi, A., Magnus, W., Oberhettinger, F., and Tricomi, F. G., Tables of Integral Transforms, Vol. 1 (McGraw-Hill Book Co., Inc., New York 1954).

ET II Erdélyi, A., Magnus, W., Oberhettinger, F., and Tricomi, F. G., Tables of Integral Transforms, Vol. 2 (McGraw-Hill Book Co., Inc., New York 1954).

GW Gröbner, W., and Hofreiter, N., Integraltafel, Teil 2, Bestimmte Integrale (Springer-Verlag, New York 1966).

LU Luke, Y. L., Integrals of Bessel Functions (McGraw-Hill Book Co., Inc., New York 1962).

MV Montroll, E. W., Theory of the Vibration of Simple Cubic Lattices with Nearest Neighbor Interactions, Proceedings of the Third Berkeley Symposium on Mathematical Statistics and Probability, edited by Neyman, J., Vol. 3, Astronomy and Physics (University of California Press, Berkeley 1956).

NM Nakagami, M., Statistical Studies on Fading, Diversity Effects and Characteristics of Diversity Receiving Systems, in Japanese (Shukyo-sha Book Co., Inc., Tokyo 1947).

OB Oberhettinger, F., Tables of Bessel Transforms (Springer-Verlag, New York 1972).

OL Oberhettinger, F., and Badii, L., Tables of Laplace Transforms (Springer-Verlag, New York 1973).

WA Watson, G. N., A Treatise on the Theory of Bessel Functions, second edition (Cambridge University Press, London 1966).

\section{Supplementary References}

Erdélyi, A., Magnus, W., Oberhettinger, F., and Tricomi, F. G., Higher Transcendental Functions, Vols. 1 and 2 (McGrawHill Book Co., Inc., New York 1953).

Gradshteyn, I. S., and Ryzhik, I. M., Table of Integrals, Series, and Products, translation edited by Jeffrey, A. (Academic Press, New York 1965).

Magnus, W., Oberhettinger, F., and Soni, R. P., Formulas and Theorems for the Special Functions of Mathematical Physics (Springer-Verlag, New York 1966).

(Paper 78B3-408) 